\title{
LOW COMPLEXITY FULL DUPLEX MIMO SYSTEMS: ANALOG CANCELER ARCHITECTURES, BEAMFORMING DESIGN, AND FUTURE DIRECTIONS
}

\author{
George C. Alexandropoulos \\ Department of Informatics and Telecommunications, National and Kapodistrian University of Athens \\ Panepistimiopolis Ilissia, 15784 Athens, Greece
}

NOTE: Corresponding author: George C. Alexandropoulos, alexandg@di.uoa.gr

\begin{abstract}
The hardware complexity of the analog Self-Interference (SI) canceler in conventional full duplex Multiple Input Multiple Output (MIMO) designs mostly scales with the number of transmit and receive antennas, thus exploiting the benefits of analog cancellation becomes impractical for full duplex MIMO transceivers, even for a moderate number of antennas. In this paper, we provide an overview of two recent hardware architectures for the analog canceler comprising of reduced number of cancellation elements, compared to the state of the art, and simple multiplexers for efficient signal routing among the transceiver radio-frequency chains. The one architecture is based on analog taps and the other on AUXiliary (AUX) Transmitters (TXS). In contrast to the available analog cancellation architectures, the values for each tap or each AUX TX and the configuration of the multiplexers are jointly designed with the digital transceiver beamforming filters according to desired performance objectives. We present a general optimization framework for the joint design of analog SI cancellation and digital beamforming, and detail an example algorithmic solution for the sum-rate optimization objective. Our representative computer simulation results demonstrate the superiority, both in terms of hardware complexity and achievable performance, of the presented low complexity full duplex MIMO schemes over the relative available ones in the literature. We conclude the paper with a discussion on recent simultaneous transmit and receive operations capitalizing on the presented architectures, and provide a list of open challenges and research directions for future FD MIMO communication systems, as well as their promising applications.
\end{abstract}

Keywords - Analog cancellation, beamforming, full duplex, MIMO, self-interference modeling, simultaneous transmit and receive

\section{INTRODUCTION}

In band full duplex, also known shortly as Full Duplex (FD), is a candidate technology for sixth Generation (6G) wireless systems because of the potential spectral efficiency gains and latency improvements that can be achieved through Simultaneous Transmit And Receive (STAR) operations within the entire frequency band [1, 2 , 3]. An FD radio can transmit and receive at the same time and same frequency resource unit, consequently, it can offer increased spectrum usage flexibility compared to a half duplex radio. Current wireless systems exploit Multiple Input Multiple Output (MIMO) communications, where increasing the number of transmit and receive antennas can increase the spatial Degrees of Freedom (DoF), hence boosting spectral efficiency and link reliability. Combining FD with MIMO communications can provide further spectral efficiency gains $[4,5,6,7,8,9,10]$. Thus, enabling FD MIMO technology, for small to large antenna array systems, is of high interest in order to achieve the demanding throughput, latency, and sensing requirements of fifth Generation (5G) and beyond wireless communication systems $[11,12]$.

An FD radio suffers from Self Interference (SI), which is the signal transmitted by the FD radio Transmitter (TX) that leaks to the FD radio Receiver (RX). At the RX of the FD radio, the power of the SI signal can be many times stronger than the power of the received signal of interest (which is transmitted from another radio). Consequently, SI can severely degrade the reception of the signal of interest, and thus SI mitigation is required in order to maximize the spectral efficiency gain of the FD operation. As the number of antennas increases, mitigating SI becomes more challenging, since more antennas naturally result in more SI components. For the case of a Single Input Single Output (SISO) FD node, it has been demonstrated $[13,14]$ that significant SI mitigation can be achieved via a combination of Analog and Digital (A/D) cancellation techniques, where an estimate of the received SI is subtracted from the received signal (which is the sum of the SI signal and signal of interest). A straightforward extension of SI mitigation solutions used in SISO FD to the case of MIMO FD can be envisioned. However, the hardware resources required for analog SI cancellation become the main bottleneck, since they scale with the number of antenna elements. Specifically, for the two most widely considered analog canceler solutions, which are: $i$ ) the architecture based on taps (a tap consists of analog components that implement delay, phase shift, and attenuation) $[13,15]$; and ii) the architecture based on AUXiliary (AUX) TX Radio-Frequency (RF) chains (an AUX TX $\mathrm{RF}$ chain generates an analog cancellation signal from an input digital reference signal) $[14,16]$, the hardware 
requirements in MIMO scenarios are as follows. For the case where the analog canceler is based on multiple taps, an extension to MIMO requires at least $M_{k} N_{k}$ taps with $M_{k}$ and $N_{k}$ denoting the number of RX and TX antennas, respectively, at an FD MIMO node $k$. For the case where the analog canceler is based on multiple AUX TX RF chains, an extension to MIMO requires at least $M_{k}$ AUX TXs. Consequently, depending on the number of TX and RX antennas at the FD MIMO node, the extension of SISO analog canceler solutions to the MIMO case may be prohibitively complex. Thus, some works have proposed only digital SI mitigation for FD MIMO [4, 7]. These approaches exploit the availability of multiple antennas at the FD node in order to provide SI mitigation via digital BeamForming (BF); such an approach is known as spatial suppression. However, as has been pointed out, spatial suppression approaches often result in lower rates for both the outgoing and incoming signals of interest, since some of the available spatial DoF are solely devoted for mitigating SI.

In this paper, we present an overview of the recent architectures of $[17,18,19]$ for analog SI cancellation in FD MIMO systems and present an optimization framework for jointly designing the analog canceler and the $\mathrm{TX} / \mathrm{RX}$ digital BF parameters. The first architecture for analog cancellation consists of multi-tap hardware, where the number of taps does not increase with the number of TX or RX antenna elements. The second architecture includes AUX TX RF chains whose number does not again depend on the number of TX or RX antennas. The number of taps in the one architecture and that of AUX TXs in the other can be chosen offline as a function of size constraints, cost per tap and cost per AUX TX RF chain, or other constraints on the analog canceler hardware. Both simplified analog canceler architectures are enabled via the use of MUltipleXers (MUXs) and DEMUltipleXers (DEMUXs), which allow flexible connectivity between the taps or AUX TXs and the transceiver antennas. The settings of taps or AUX TXs and the configurations of MUXs/DEMUXs is computed via the presented optimization framework. The flexible signal routing via MUXs/DEMUXs enables the use of reduced taps or AUX TXs in an optimized way, since either of the latter will be used between the subset of TX and RX antennas where they are mostly beneficial. The digital beamformer and analog canceler parameters are thus designed by taking into account each other's capabilities, hence, the burden of SI mitigation is split between digital BF and analog cancellation. We note that the related work [4] has considered joint design of digital BF and analog cancellation, however these and related solutions $[20,21]$ assume underlying analog canceler hardware as in $[13,6,15,16,14]$, which scales with the number of transceiver antennas. For the JointNull solution proposed in [22], although the number of analog cancelers does not necessarily scale with the number of antennas, the underlying architecture of the canceler (i.e., number of taps or AUX TXs) is not explicitly taken into account in the BF design. As our simulation results will show, the reviewed analog canceler architectures together with the presented joint design of analog cancellation and TX/RX digital BF are capable of achieving higher rates with less hardware compared to State-of-the-Art (SotA) FD MIMO solutions. This paper's contributions can be summarized as follows.

- We provide a detailed overview of two recent analog SI canceler architectures, one based on multiple taps and another one consisting of multiple AUX TX RF chains. Both architectures include networks of MUXs/DEMUXs intended for efficient signal routing between either the taps or AUX TXs and the transceiver antennas.

- We present a general optimization framework for the joint design of analog SI cancellation and digital transceiver BF at FD MIMO nodes.

- We present an example algorithmic design for the analog cancellation parameters as well as the digital TX precoder and RX combiner that targets the maximization of the FD sum rate performance.

- Extensive simulation results incorporating realistic models for non-ideal hardware for both cosidered analog canceler architectures are presented. We compare both designed low complexity FD MIMO schemes with lately available ones in terms of hardware complexity and achievable performance.

- We discuss recent STAR schemes capitalizing on the presented FD MIMO hardware architectures, and present a list of open challenges and research directions for future FD MIMO communication systems, as well as their promising applications.

The outline of the paper is as follows. The considered system and signal models are included in Section 2, whereas Section 3 details two analog SI cancellation architectures. Our general optimization framework for FD MIMO operation is provided in Section 4, and Section 5 presents an example design optimization formulation together with a detailed low complexity solution. Simulation results are presented and explained in Section 6, while Section 7 discusses recent applications of the considered FD MIMO architectures together with open research challenges for FD systems. The paper is concluded in Section 8.

Notation: Vectors and matrices are denoted by boldface lowercase and boldface capital letters, respectively. The transpose and Hermitian transpose of $\mathbf{A}$ are denoted by $\mathbf{A}^{\mathrm{T}}$ and $\mathbf{A}^{\mathrm{H}}$, respectively, and $\operatorname{det}(\mathbf{A})$ is the determinant of $\mathbf{A}$, while $\mathbf{I}_{n}(n \geq 2)$ is the $n \times n$ identity matrix and $\mathbf{0}_{m \times n}(m \geq 2$ and $n \geq 1)$ represents the $m \times n$ matrix with all zeros. $\|\mathbf{a}\|$ stands for the Euclidean norm of a, operand $\odot$ represents the Hadamard entry-wise product, and $\operatorname{diag}\{\mathbf{a}\}$ denotes a square diagonal matrix with a's elements in its main diagonal. $[\mathbf{A}]_{i, j},[\mathbf{A}]_{(i,:)}$, and $[\mathbf{A}]_{(:, j)}$ represent A's $(i, j)$-th element, $i$-th row, and $j$-th column, respectively, while $[\mathbf{a}]_{i}$ denotes the $i$-th element of $\mathbf{a}$. $\mathbb{R}$ 
and $\mathbb{C}$ represent the real and complex number sets, respectively, $\mathbb{E}\{\cdot\}$ is the expectation operator, and $|\cdot|$ denotes the amplitude of a complex number.

\section{SYSTEM AND SIGNAL MODELS}

We consider a wireless communication system comprising of an FD MIMO node $k$ that wishes to communicate concurrently with a multi-antenna node $q$ in the downlink and a multi-antenna node $m$ in the uplink, as shown in Fig. 1. We focus on investigating efficient FD operation at a single node, as such, we henceforth assume without loss of generality that nodes $q$ and $m$ operate in half duplex mode.

Suppose that the FD MIMO node $k$ in Fig. 1 is equipped with $N_{k}$ TX antenna elements and $M_{k}$ RX antenna elements. Each antenna element is attached to a dedicated TX RF chain, and similarly holds for the RX antenna elements and their respective RF chains. A TX RF chain consists of a Digital to Analog Converter (DAC), a mixer which upconverts the signal from the baseband to the RF, and a Power Amplifier (PA). An RX RF chain consists of a Low Noise Amplifier (LNA), a mixer which downconverts the signal from the RF to the baseband, and an Analog to Digital Converter (ADC). At the TX side, upsample and pulse shape processing are used to prepare the baseband signal for DAC sampling and RF transmission. At the RX side, a corresponding matched filter and downsampling is performed. The half duplex multi-antenna nodes $q$ and $m$ are assumed to have $M_{q}$ and $N_{m}$ antennas, respectively, with each antenna connected to a respective RF chain.

For presentation clarity purposes, we assume narrowband flat fading channels for our signal model. The extensions for wideband frequency selective channels is the focus of ongoing works (e.g., [23]). All nodes are considered capable of performing digital BF; for simplicity, we assume hereinafter that digital TX and RX BF at the focused FD MIMO node $k$ is realized with linear filters. In particular, we assume that node $k$ makes use of the precoding matrix $\mathbf{V}_{k} \in \mathbb{C}^{N_{k} \times d_{k}}$ for processing its unit power symbol vector $\mathbf{s}_{k} \in \mathbb{C}^{d_{k} \times 1}$ (chosen from a discrete modulation set) before transmission. The dimension of $\mathbf{s}_{k}$ satisfies $d_{k} \leq \min \left\{M_{q}, N_{k}\right\}$, which complies with the available spatial DoF for the downlink $M_{q} \times$ $N_{k}$ MIMO channel. Similarly, node $m$ processes its unit power symbol vector $\mathbf{s}_{m} \in \mathbb{C}^{d_{m} \times 1}$ (chosen again from a discrete modulation set) with a precoding matrix $\mathbf{V}_{m} \in$ $\mathbb{C}^{N_{m} \times d_{m}}$, where $d_{m} \leq \min \left\{M_{k}, N_{m}\right\}$. Both the downlink and uplink transmissions are power-limited according to $\mathbb{E}\left\{\left\|\mathbf{V}_{k} \mathbf{s}_{k}\right\|^{2}\right\} \leq \mathrm{P}_{k}$ and $\mathbb{E}\left\{\left\|\mathbf{V}_{m} \mathbf{s}_{m}\right\|^{2}\right\} \leq \mathrm{P}_{m}$, respectively. Following the above definitions, the baseband received signal $\mathbf{y}_{q} \in \mathbb{C}^{M_{q} \times 1}$ at the half duplex node $q$ can be mathematically expressed as:

$$
\mathbf{y}_{q} \triangleq \mathbf{H}_{q, k} \mathbf{V}_{k} \mathbf{s}_{k}+\mathbf{H}_{q, m} \mathbf{V}_{m} \mathbf{s}_{m}+\mathbf{n}_{q}
$$

where $\mathbf{H}_{q, k} \in \mathbb{C}^{M_{q} \times N_{k}}$ is the downlink channel matrix (i.e., between nodes $q$ and $k$ ), $\mathbf{H}_{q, m} \in \mathbb{C}^{M_{q} \times N_{m}}$ denotes the channel matrix for inter-node interference (i.e., between nodes $q$ and $m$ ) , and $\mathbf{n}_{q} \in \mathbb{C}^{M_{q} \times 1}$ represents the Additive White Gaussian Noise (AWGN) vector at node $q$ with covariance matrix $\sigma_{q}^{2} \mathbf{I}_{M_{q}}$.

Upon signal reception at the FD MIMO node $k$, analog SI cancellation is first applied to the signals received at its $\mathrm{RX}$ antenna elements before these signals enter the RX RF chains, as shown in Fig. 1. Notice that the output of the analog canceler is added to the received signals before their input to the RX RF chains. We utilize the notation $\mathbf{C}_{k} \in \mathbb{C}^{M_{k} \times N_{k}}$ to represent the signal processing realized by the analog canceler. Depending on the deployed hardware components, the analog canceler can have as inputs analog or digital signals. In Section 3, we will detail the hardware characteristics of our two analog canceler architectures. We will also show that for both architectures, the baseband representation for the output signal of the analog canceler at the FD node $k$, which we label as $\tilde{\mathbf{y}}_{k} \in \mathbb{C}^{M_{k} \times 1}$, is given by:

$$
\tilde{\mathbf{y}}_{k} \triangleq \mathbf{C}_{k} \mathbf{V}_{k} \mathbf{s}_{k} .
$$

By assuming that the digitally converted and downsampled output signals of the RX RF chains at node $k$ are linearly processed in the baseband by the combining matrix $\mathbf{U}_{k} \in \mathbb{C}^{d_{m} \times M_{k}}$, the estimated symbol vector $\hat{\mathbf{s}}_{m} \in \mathbb{C}^{d_{m} \times 1}$ for $\mathbf{s}_{m}$ is derived as:

$$
\widehat{\mathbf{s}}_{m} \triangleq \mathbf{U}_{k}\left(\mathbf{y}_{k}+\overline{\mathbf{y}}_{k}+\tilde{\mathbf{y}}_{k}+\mathbf{n}_{k}\right),
$$

where the complex-valued $M_{k}$-element vectors $\mathbf{y}_{k}$ and $\overline{\mathbf{y}}_{k}$ are the baseband representations of the received signal of interest and received SI signal, respectively, at node $k$. In addition, $\mathbf{n}_{k} \in \mathbb{C}^{M_{k} \times 1}$ denotes the received AWGN vector at node $k$ with covariance matrix $\sigma_{k}^{2} \mathbf{I}_{M_{k}}$. The vector $\mathbf{y}_{k}$ in (3) is given by:

$$
\mathbf{y}_{k} \triangleq \mathbf{H}_{k, m} \mathbf{V}_{m} \mathbf{s}_{m}
$$

where $\mathbf{H}_{k, m} \in \mathbb{C}^{M_{k} \times N_{m}}$ is the uplink channel matrix (i.e., between nodes $k$ and $m$ ), while $\mathbf{y}_{k}$ is obtained as:

$$
\overline{\mathbf{y}}_{k} \triangleq \mathbf{H}_{k, k} \mathbf{V}_{k} \mathbf{s}_{k}
$$

with $\mathbf{H}_{k, k} \in \mathbb{C}^{M_{k} \times N_{k}}$ denoting the SI channel seen at the $\mathrm{RX}$ antennas of node $k$ due to its own downlink transmission.

For cases where the residual SI signal in (3) (i.e., after performing analog cancellation and TX/RX digital $\mathrm{BF}$ ) is above the noise floor, further digital SI mitigation [24, 25] can be applied on the signal $\hat{\mathbf{s}}_{m}$ to bring the residual interference below that floor. In this paper, we focus on analyzing the combined effect of analog cancellation and TX/RX digital BF, hence, we do not model a digital SI cancellation stage.

\section{ANALOG CANCELER ARCHITECTURES}

In this section, we present in detail the hardware components of our two analog SI canceler architectures introduced in $[17,18,19]$. The first architecture is based on 


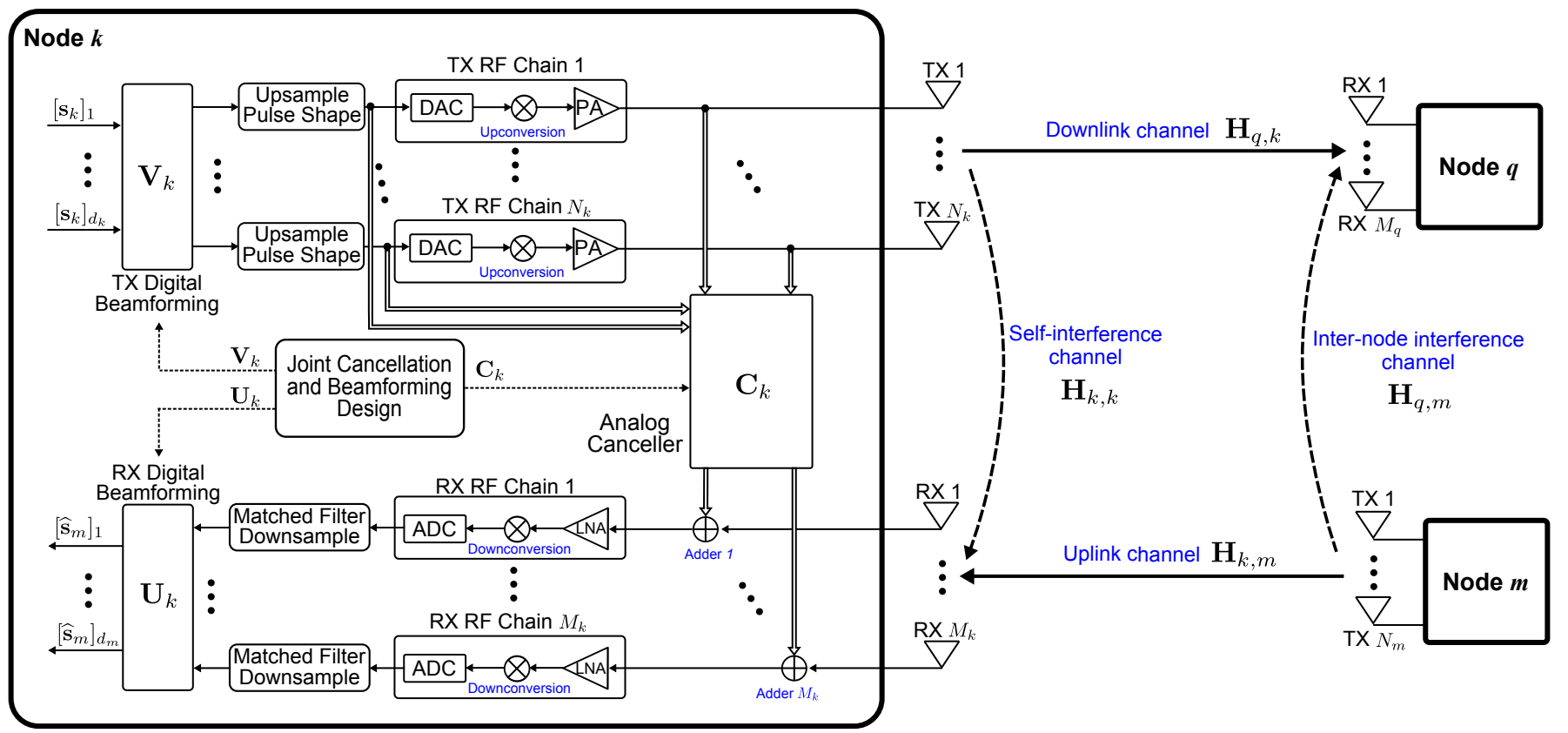

Fig. 1 - Schematic diagram of the considered system model and the proposed FD MIMO architectural components. The FD MIMO node $k$ communicates with the two half duplex multi-antenna nodes $q$ and $m$, the former in the downlink and the latter in the uplink communications. Node $k$ incorporates processing blocks dedicated to TX and RX digital BF, analog SI cancellation, as well as to the joint design of analog cancellation and TX/RX digital BF.

the utilization of analog taps and is thus labeled as multitap canceler. The second architecture consists of AUX TXs and termed as multi-AUX-TX canceler. The joint design of the analog canceler parameters and TX and RX digital BF will be detailed in the following sections.

\subsection{Multi-tap analog canceler architecture}

The hardware components of the multi-tap canceler, initially introduced in $[17,19]$, for the FD MIMO node $k$ are illustrated in Fig. 2. In this figure, $N \leq M_{k} N_{k}$ canceler taps are applied via MUXs to the outputs of the TX RF chains and via DEMUXs and adders to the inputs of the RX RF chains. One way of implementing ana$\log$ RF MUXs/DEMUXs is through RF switches. With the term 'tap' we denote a fixed delay-variable phase shiftervariable attenuator line, as considered in [15]. It is shown in Fig. 2 that the input of each analog canceler tap is connected to a corresponding $N_{k}$-to-1 MUX which allows routing of any of the $N_{k}$ TX RF chain signals to the input of the tap. The connection from each TX RF chain to each MUX input can be done via power dividers or directional couplers [15]. The signal that inputs to a tap undergoes a delay, phase shift, and attenuation, and this generates as an output an analog cancellation signal. The output of each tap is connected to a 1-to- $M_{k}$ DEMUX, which routes the cancellation signal at the output of the tap to one of the adders located just before the RX RF chains. There is a total of $M_{k} N$ such adders and we use "Adder $i, j$ " to label the adder that connects DEMUX $j$ to $\mathrm{RX} \operatorname{RF}$ chain $i$. Thus, the signal input to the $i$-th RX RF chain is the result of adding $N$ cancellation signals to the signal received at the $i$-th RX antenna element. Since the adders are connected to DEMUXs, some of the adders may have zero in one of the inputs depending on the DEMUXs' settings. The adders before the RX RF chains can be implemented via power combiners or directional couplers.

As illustrated in Fig. 2, analog SI cancellation is applied to the signals received at the RX antenna elements before these signals enter the RX RF chains. Recall from Section 2 that we utilize the notation $\mathbf{C}_{k} \in \mathbb{C}^{M_{k} \times N_{k}}$ to represent the signal processing realized by the analog canceler. Thus, for the multi-tap canceler architecture in Fig. 2, $\mathbf{C}_{k}$ captures the configuration of the MUXs/DEMUXs and the canceler tap values. We model $\mathbf{C}_{k}$ in baseband representation as the following cascade of three matrices:

$$
\mathbf{C}_{k} \triangleq \mathbf{L}_{3} \mathbf{L}_{2} \mathbf{L}_{1}
$$

where $\mathbf{L}_{1} \in \mathbb{R}^{N \times N_{k}}, \mathbf{L}_{2} \in \mathbb{C}^{N \times N}$, and $\mathbf{L}_{3} \in \mathbb{R}^{M_{k} \times N}$. The elements $\left[\mathbf{L}_{1}\right]_{i, j}$ with $i=1,2, \ldots, N$ and $j=1,2, \ldots, N_{k}$, and $\left[\mathbf{L}_{3}\right]_{i, j}$ with $i=1,2, \ldots, M_{k}$ and $j=1,2, \ldots, N$ take the binary values 0 or 1 , and it must hold that:

$$
\begin{aligned}
& \sum_{j=1}^{N_{k}}\left[\mathbf{L}_{1}\right]_{i, j}=1 \forall i=1,2, \ldots, N, \\
& \sum_{i=1}^{M_{k}}\left[\mathbf{L}_{3}\right]_{i, j}=1 \forall j=1,2, \ldots, N .
\end{aligned}
$$

The $i$-th row of $\mathbf{L}_{1}$ indicates the MUX configuration at the input of the $i$-th tap of the canceler, while the $i$-th column of $\mathbf{L}_{3}$ shows the DEMUX configuration at the output of the $i$-th tap of the canceler. The $\mathbf{L}_{2}$ in (6) is a diagonal matrix whose complex entries represent the attenuation and 


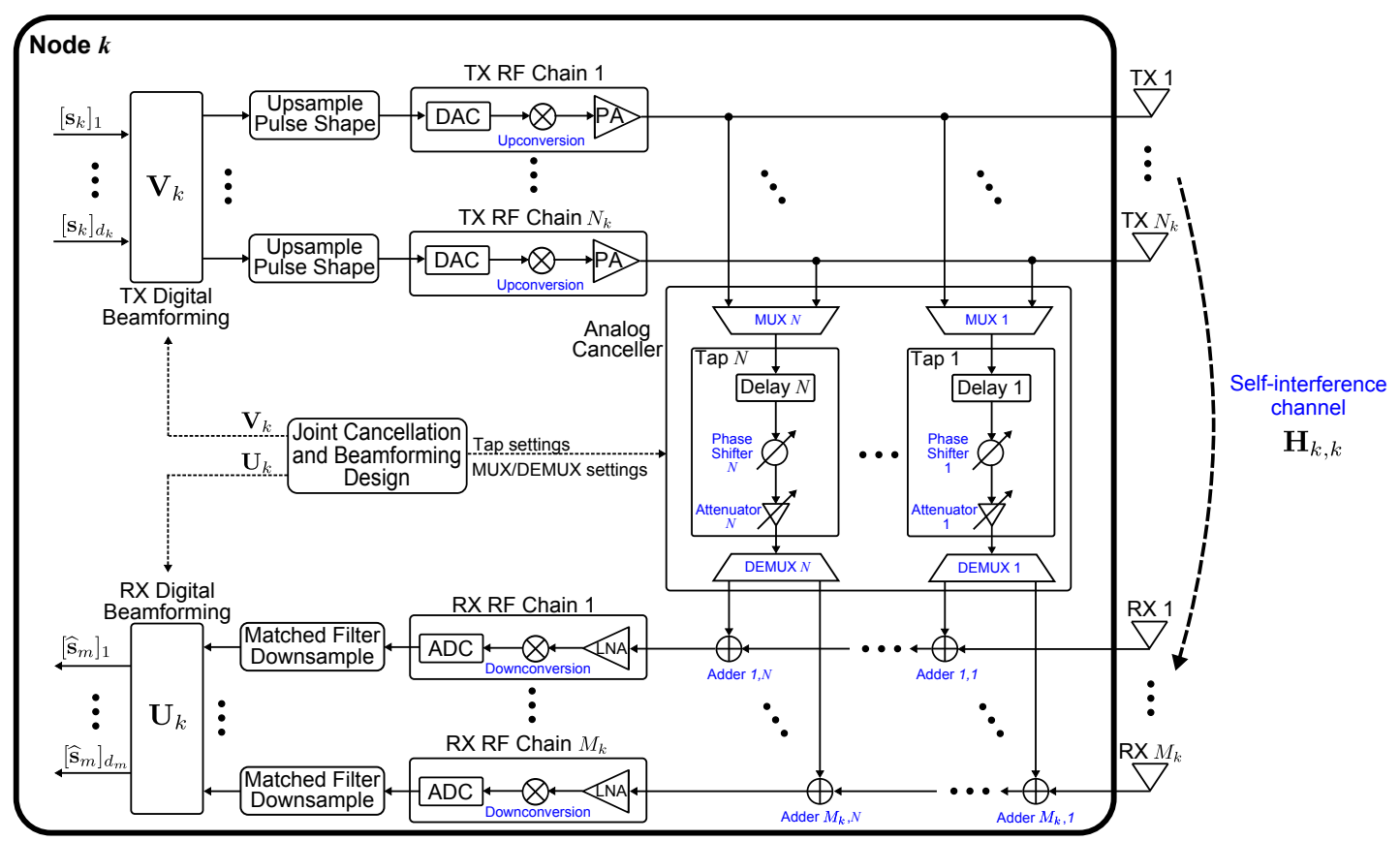

Fig. 2 - The FD MIMO architecture at node $k$ with the multi-tap analog canceler. This canceler consists of $N$ taps, which are connected via MUXs to the outputs of the TX RF chains and via DEMUXs and adders to the inputs of the RX RF chains. With the term "tap" we denote a line of fixed delay, variable phase shifter, and attenuator.

phase shift of the canceler taps; particularly, the magnitude and phase of the element $\left[\mathbf{L}_{2}\right]_{i, i}$ with $i=1,2, \ldots, N$ specify the attenuation and phase of the $i$-th tap. Recall that the tap delays in each canceler tap are fixed and since we focus on a narrowband system, we model the effects of the $i$-th tap delay as a phase shift that is incorporated to the phase of $\left[\mathbf{L}_{2}\right]_{i, i}$.

The adoption of MUXs/DEMUXs for signal routing is a distinct feature of our multi-tap analog SI canceler. The flexible signal routing that is enabled by the MUXs/DEMUXs allows the use of a reduced number of taps for analog cancellation, compared to the number of taps required by the designs in $[6,13,15]$, which require at least one tap between each TX RF chain and each RX RF chain, hence, at least $M_{k} N_{k}$ taps. For our proposed multi-tap canceler design, the total number of taps $N \leq M_{k} N_{k}$ is flexible and can be chosen offline as a function of node size constraints, cost per tap, or other constraints on the analog canceler hardware. Furthermore, the TX and RX digital beamformers and analog canceler will adapt to each other's capabilities via our proposed joint design of analog cancellation and digital BF, which will be explained in Section 4 .

\subsection{Multi-AUX-TX analog canceler architec- ture}

Fig. 3 depicts the hardware components of the multiAUX-TX canceler, firstly introduced in $[18,19]$, for the FD MIMO node $k$. The analog cancellation signal is generated through $N \leq M_{k}$ AUX TXs, which are connected via DEMUXs and adders to the RX RF chains. An AUX TX is a
TX RF chain that is used locally to generate the cancellation signal; as such, the AUX TX does not require a PA. The input to the $N$ AUX TX RF chains is generated in the digital domain and is obtained from a linear transformation of the $N_{k}$ output signals of the TX digital beamformer. We represent this linear transformation of the transmitted signal to generate locally the cancellation signal by the matrix $\mathbf{L}_{4} \in \mathbb{C}^{N \times N_{k}}$. It is emphasized that in the multi-AUX-TX architecture a copy of the SI signal is fed to the analog canceler in the digital domain, whereas in the multi-tap architecture depicted in Fig. 2 this connection takes place in the analog domain. However, the analog canceler outputs an analog signal for both proposed architectures. The output of each AUX TX feeds a corresponding DEMUX whose role is to route its input signal to one of the $M_{k}$ adders it is attached to. The latter mechanism is analogous to the DEMUX and adder connections of the multi-tap canceler described in Section 3.1. The baseband representation of the signal processing realized by the multi-AUX-TX canceler is modeled similar to the multi-tap case by the matrix $\mathbf{C}_{k} \in \mathbb{C}^{M_{k} \times N_{k}}$, which is now given by the following expression:

$$
\mathbf{C}_{k} \triangleq \mathbf{L}_{5} \mathbf{L}_{4}
$$

where $\mathbf{L}_{5} \in \mathbb{R}^{M_{k} \times N}$. The $i$-th column of $\mathbf{L}_{5}$ indicates the configuration of the DEMUX connected to the $i$-th AUX TX RF chain. Thus, the elements $\left[\mathbf{L}_{5}\right]_{i, j}$ with $i=1,2, \ldots, M_{k}$ and $j=1,2, \ldots, N$ take the binary values 0 or 1 , and it must hold that:

$$
\sum_{i=1}^{M_{k}}\left[\mathbf{L}_{5}\right]_{i, j}=1 \forall j=1,2, \ldots, N .
$$




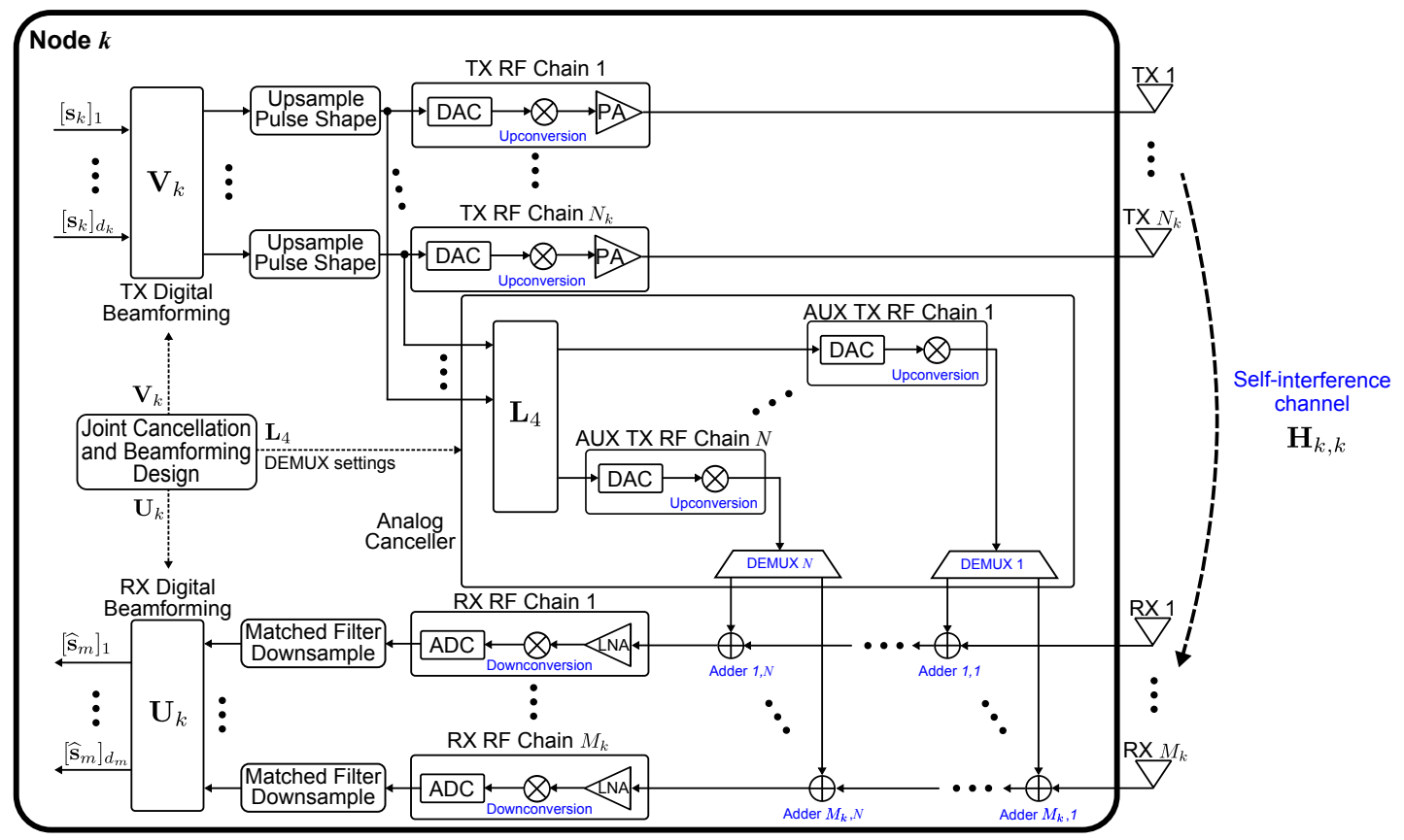

Fig. 3 - The FD MIMO architecture at node $k$ with the multi-AUX-TX analog canceler. The analog canceler consists of $N$ AUX TX RF chains that locally generate the cancellation signal, which are connected via DEMUXs and adders to the inputs of the RX RF chains.

The flexible routing of the outputs of the AUX TXs via DEMUXs that enables adjustable processing of the SI signal is a distinct feature of our multi-AUX-TX canceler. The designs $[16,14]$ that adopt AUX TX RF chains do not include DEMUXs and utilize one AUX TX RF chain per RX RF chain (e.g., $M_{k}$ AUX TX RF chains will be needed for node $k$ with the designs $[14,16])$. This means that if the number of RX $\mathrm{RF}$ chains increases, the hardware required for the analog SI canceler increases as well. In contrast, our proposed multi-AUX-TX architecture can have any number $N \leq M_{k}$ of AUX TXs, and the effective use of the available AUX TX $\mathrm{RF}$ chains will be handled via the joint design of analog cancellation and digital BF, which will be detailed in the following section.

\section{PROPOSED FD MIMO OPTIMIZATION FRAMEWORK}

In this section, we present an FD MIMO optimization framework for the joint design of the hardware components of our analog canceler architectures described in Section 3 together with the TX and RX digital BF blocks, as included in our system model in Fig. 1, in order to satisfy certain performance objectives. Capitalizing on the signal model introduced in Section 2, we are particularly interested in the joint design of the analog canceler matrix $\mathbf{C}_{k}$, the digital precoding matrix $\mathbf{V}_{k}$, and the digital combining matrix $\mathbf{U}_{k}$ for the FD MIMO node $k$. We define the general objective function $f(\cdot)$ having as inputs the latter matrices and representing either a sole scalar performance objective, such as the average sum throughput of the FD MIMO operation, or a multi-objective performance function [26], like the average sum throughput together with an energy efficiency metric. Our general optimization framework for the joint design of $\mathbf{C}_{k}, \mathbf{V}_{k}$, and $\mathbf{U}_{k}$ at node $k$ is mathematically expressed by the following general optimization problem ${ }^{1}$ :

$$
\begin{array}{rl}
\mathcal{O P}: \max _{\mathbf{C}_{k}, \mathbf{V}_{k}, \mathbf{U}_{k}} & f\left(\mathbf{C}_{k}, \mathbf{V}_{k}, \mathbf{U}_{k}\right) \\
\text { s.t. } & \operatorname{tr}\left\{\mathbf{V}_{k} \mathbf{V}_{k}^{\mathrm{H}}\right\} \leq \mathrm{P}_{k}, \\
& \text { Constraints on } \mathbf{C}_{k} \text { structure, } \\
& g_{1}\left(\left(\mathbf{H}_{k, k}+\mathbf{C}_{k}\right) \mathbf{V}_{k} \mathbf{s}_{k}\right) \leq \boldsymbol{\lambda}_{\mathrm{A}}, \\
& g_{2}\left(\mathbf{U}_{k}\left(\mathbf{H}_{k, k}+\mathbf{C}_{k}\right) \mathbf{V}_{k} \mathbf{s}_{k}\right) \leq \boldsymbol{\lambda}_{\mathrm{D}},
\end{array}
$$

where constraint (C1) relates to the total transmit power budget at node $k$ and constraint (C2) refers to the hardware capabilities of the analog canceler, which impose certain limitations on the construction of $\mathbf{C}_{k}$. It follows from the discussion in Section 3.1 that (C2) for the proposed multi-tap canceler architecture specifies to:

$$
\begin{aligned}
\mathbf{C}_{k} & =\mathbf{L}_{3} \mathbf{L}_{2} \mathbf{L}_{1} \text { with (7a), (7b), and } \\
{\left[\mathbf{L}_{2}\right]_{i, j} } & =0 \text { for } i, j=1,2, \ldots, N \text { with } i \neq j,
\end{aligned}
$$

\footnotetext{
${ }^{1}$ The proposed optimization framework focuses on the joint design of the core processing blocks at the FD MIMO node $k$ for a given power budget $\mathrm{P}_{k}$, without considering the processing at nodes $q$ and $m$. A more general problem formulation for the considered system would include in the joint optimization the power allocation between downlink and uplink as well as the RX combining at node $q$ and the TX precoding of node $m$. However, in this paper, we study FD MIMO operation at node $k$ with conventional downlink and uplink control communications, and we leave the more general joint optimization that would require additional control phases for the communication of the optimized parameters as future work.
} 
whereas, for the multi-AUX-TX canceler architecture, (C2) can be expressed using the description of Section 3.2 as:

$$
\mathbf{C}_{k}=\mathbf{L}_{5} \mathbf{L}_{4} \text { with (9). }
$$

In addition, constraint (C3) including the general vector function $g_{1}: \mathbb{C}^{M_{k} \times 1} \rightarrow \mathbb{R}_{+}^{M_{k} \times 1}$ sets the threshold values inside the vector $\lambda_{\mathrm{A}} \in \mathbb{R}_{+}^{M_{k} \times 1}$ on functions of the instantaneous residual SI appearing at the $M_{k} \mathrm{RX}$ antenna elements after analog cancellation and before the RX RF chains. Two examples of the function $g_{1}$ are: $i$ ) the element-wise instantaneous powers of the residual SI signals; and ii) their summation. For the former $g_{1}$ example, (C3) results to $\left|\left[\left(\mathbf{H}_{k, k}+\mathbf{C}_{k}\right) \mathbf{V}_{k} \mathbf{s}_{k}\right]_{i}\right|^{2} \leq\left[\boldsymbol{\lambda}_{\mathrm{A}}\right]_{i}$ with $i=1,2, \ldots, M_{k}$, whereas for the latter example $g_{1}\left(\left(\mathbf{H}_{k, k}+\mathbf{C}_{k}\right) \mathbf{V}_{k} \mathbf{s}_{k}\right)=\left\|\left(\mathbf{H}_{k, k}+\mathbf{C}_{k}\right) \mathbf{V}_{k} \mathbf{s}_{k}\right\|^{2}$ and consequently $\boldsymbol{\lambda}_{\mathrm{A}} \equiv \lambda_{\mathrm{A}} \in \mathbb{R}_{+}$. Finally, constraint (C4) with the general vector function $g_{2}: \mathbb{C}^{d_{m} \times 1} \rightarrow \mathbb{R}_{+}^{d_{m} \times 1}$ imposes the values included in the vector $\boldsymbol{\lambda}_{\mathrm{D}} \in \mathbb{R}_{+}^{d_{m} \times 1}$ on functions of the $d_{m}$ instantaneous residual SI signals obtained after applying analog cancellation and RX digital combining. Similar to $g_{1}$, instances of the function $g_{2}$ are the individual instantaneous powers of the latter $d_{m}$ signals as well as their summation.

The main novel components of the proposed FD MIMO optimization framework in $\mathcal{O P}$ can be summarized as follows. First, the digital TX and RX BF design takes into explicit account the available number of analog taps $N$, or number of AUX TXs $N$, of the analog SI cancellation block. Although some available BF solutions [4, 20, 22] for FD MIMO systems consider the presence of an ana$\log$ SI canceler, the details of its hardware limitations are excluded from the BF design. Second, the proposed FD MIMO framework is the only one that explicitly considers the case where $N<\min \left\{M_{k}, N_{k}\right\}$, i.e., the available number of analog taps, or AUX TX RF chains, may be smaller than both the numbers of TX and RX RF chains. This is an important feature for practical FD MIMO deployments, since current analog SI cancellation solutions require either very large numbers of taps, of the order of $M_{k} N_{k}$ for the architecture proposed in [6], or very large number of AUX TXs, of the order of $M_{k}$ for the architecture presented in [16]. Third, our framework has the advantage of a more improved utilization of the spatial DoF offered by the available multiple antennas at the FD MIMO node $k$. For example, if the analog canceler consists of only $N=1$ tap, or $N=1$ AUX TX, then its cancellation capabilities are very limited, and more spatial DoF need to be devoted from the TX and RX BF blocks for meeting the thresholds $\lambda_{\mathrm{A}}$ and $\boldsymbol{\lambda}_{\mathrm{D}}$ in (C3) and (C4). At the other extreme, if $N$ can be afforded to be large, the digital $\mathrm{BF}$ design may exploit the fact that a significant part of SI mitigation is handled by the analog canceler, and thus make use of more of the available spatial DoF for improving the quality of the incoming and outgoing signals of interest.

\section{AN EXAMPLE FD MIMO DESIGN}

Capitalizing on the general optimization framework for the joint design of $\mathbf{C}_{k}, \mathbf{V}_{k}$, and $\mathbf{U}_{k}$ at the FD MIMO node $k$ described in Section 4 , we hereinafter present an example joint design of analog cancellation and digital BF. We assume that there is no inter-node interference between the half duplex multi-antenna nodes $q$ and $m$ due to, for example, appropriate node scheduling $[9,10]$ for the FD operation of node $k$. Extensions considering this interference for the cases where it is known at either the receiving node $q$ and/or the transmitting node $k$, or unknown to both, are left for future works. The latter assumption translates to setting the channel matrix between the involved nodes as $\mathbf{H}_{q, m}=\mathbf{0}_{M_{q} \times N_{k}}$. For this case, the model given by (1) for the received signal at node $q$ reduces to:

$$
\mathbf{y}_{q}=\mathbf{H}_{q, k} \mathbf{V}_{k} \mathbf{s}_{k}+\mathbf{n}_{q} \text {. }
$$

We rewrite the signal model (3) that describes the estimation for $\widehat{\mathbf{s}}_{m}$ at the RX of node $k$ as:

$$
\hat{\mathbf{s}}_{m}=\mathbf{U}_{k}\left(\mathbf{H}_{k, m} \mathbf{V}_{m} \mathbf{s}_{m}+\widetilde{\mathbf{H}}_{k, k} \mathbf{V}_{k} \mathbf{s}_{k}+\mathbf{n}_{k}\right),
$$

where $\widetilde{\mathbf{H}}_{k, k} \in \mathbb{C}^{M_{k} \times N_{k}}$ denotes the effective SI channel after performing analog cancellation, which is defined as: $\widetilde{\mathbf{H}}_{k, k} \triangleq \mathbf{H}_{k, k}+\mathbf{C}_{k}$.

An important performance objective function $f(\cdot)$ for the considered system is the FD rate defined as the sum rate of the downlink and uplink communications. We therefore focus on designing $\mathbf{C}_{k}, \mathbf{V}_{k}$, and $\mathbf{U}_{k}$ via the solution of the following optimization problem:

$$
\begin{aligned}
\mathcal{O P} 1: \max _{\mathbf{C}_{k}, \mathbf{V}_{k}, \mathbf{U}_{k}} & \mathcal{R}_{\mathrm{DL}}\left(\mathbf{V}_{k}\right)+\mathcal{R}_{\mathrm{UL}}\left(\mathbf{C}_{k}, \mathbf{V}_{k}, \mathbf{U}_{k}\right) \\
\text { s.t. } & (\mathrm{C} 1),(\mathrm{C} 2), \\
& \left\|\left[\widetilde{\mathbf{H}}_{k, k} \mathbf{V}_{k}\right]_{(j,:)}\right\|^{2} \leq \lambda_{\mathrm{A}} \forall j=1,2, \ldots, M_{k}, \\
& \left\|\left[\mathbf{U}_{k}\right]_{(i,:)}\right\|^{2}=1 \forall i=1,2, \ldots, d_{m} .
\end{aligned}
$$

In the latter problem, the achievable downlink rate $\mathcal{R}_{\mathrm{DL}}$ is a function of only the digital precoding matrix $\mathbf{V}_{k}$ and is given by:

$$
\mathcal{R}_{\mathrm{DL}}\left(\mathbf{V}_{k}\right)=\log _{2}\left(\operatorname{det}\left(\mathbf{I}_{M_{q}}+\sigma_{q}^{-2} \mathbf{H}_{q, k} \mathbf{V}_{k} \mathbf{V}_{k}^{\mathrm{H}} \mathbf{H}_{q, k}^{\mathrm{H}}\right)\right) .
$$

Note that we have assumed capacity-achieving combining at node $q$ in (12), like the non-linear Minimum Mean Squared Error (MMSE) successive interference canceler [27, Chap. 2]. The uplink rate $\mathcal{R}_{\mathrm{UL}}$ in $\mathcal{O P} 1$ is a function of $\mathbf{V}_{k}$, the analog canceler matrix $\mathbf{C}_{k}$, and the digital combining matrix $\mathbf{U}_{k}$, and is derived as:

$$
\begin{aligned}
\mathcal{R}_{\mathrm{UL}}\left(\mathbf{C}_{k}, \mathbf{V}_{k}, \mathbf{U}_{k}\right)=\log _{2}( & \operatorname{det}\left(\mathbf{I}_{d_{m}}+\sigma_{k}^{-2} \mathbf{U}_{k} \mathbf{H}_{k, m}\right. \\
& \left.\left.\times \mathbf{V}_{m} \mathbf{V}_{m}^{\mathrm{H}} \mathbf{H}_{k, m}^{\mathrm{H}} \mathbf{U}_{k}^{\mathrm{H}} \mathbf{Q}_{k}^{-1}\right)\right),
\end{aligned}
$$

where $\mathbf{Q}_{k} \in \mathbb{C}^{d_{m} \times d_{m}}$ denotes the covariance matrix of the interference-plus-noise after combining at node $k$ that can be expressed as:

$$
\mathbf{Q}_{k}=\mathbf{U}_{k} \widetilde{\mathbf{H}}_{k, k} \mathbf{V}_{k} \mathbf{V}_{k}^{\mathrm{H}} \widetilde{\mathbf{H}}_{k, k}^{\mathrm{H}} \mathbf{U}_{k}^{\mathrm{H}}+\sigma_{k}^{2} \mathbf{U}_{k} \mathbf{U}_{k}^{\mathrm{H}} .
$$


Different from the downlink rate in (12), in (13) and (14) we include the considered linear combining matrix $\mathbf{U}_{k}$ which jointly with $\mathbf{V}_{k}$ and $\mathbf{C}_{k}$ we aim to optimally design. Note that in the formulation of $\mathcal{O P} 1$ we have relaxed constraint (C3) concerning the instantaneous residual SI after analog cancellation that appears in the general $\mathcal{O P}$ to an average power per RX RF chain constraint, where the average is taken over all possible transmit symbol vectors. This constraint imposes that, at the input of each of the $M_{k} \mathrm{RX}$ RF chains, the average power of the SI signal for all transmitted symbols within a coherent channel block cannot be larger than the threshold $\lambda_{\mathrm{A}}$. Notice also that in $\mathcal{O P} 1$ we have not included a constraint similar to (C4) for the residual SI signal after digital combining. Instead we have only incorporated a constraint on the norm of the rows of $\mathbf{U}_{k}$. The reason for this simplification mainly lies on $\mathcal{O P} 1$ 's sum-rate objective function. We expect that the joint design of $\mathbf{C}_{k}, \mathbf{V}_{k}$, and $\mathbf{U}_{k}$ optimizing the uplink rate will naturally result in keeping the average power of the residual SI signal after both A/D processing at an acceptable level; acceptable level is any level allowing uplink communications. Furthermore, the unity constraint on the norm of each of the rows of $\mathbf{U}_{k}$ excludes combining solutions that result in undesired amplification of the received signals (i.e., the signals from node $m$, SI, and AWGN).

We propose to tackle $\mathcal{O P} 1$ with the following two-step approach. First, as described next in Section 5.1, we consider only the downlink which is usually more rate demanding than the uplink, and obtain the pairs of $\mathbf{C}_{k}$ and $\mathbf{V}_{k}$ designs optimizing the instantaneous downlink rate while meeting their respective constraints. Then, we solve for the best pair of $\mathbf{C}_{k}$ and $\mathbf{V}_{k}$ as well as the $\mathbf{U}_{k}$ design that jointly maximize the sum-rate performance, as will be explained in Section 5.2.

\subsection{Candidate designs for $\mathbf{C}_{k}$ and $\mathbf{V}_{k}$}

We first formulate the following downlink rate maximization problem using expression (12) for the design of the analog cancellation matrix $\mathbf{C}_{k}$ and the precoding matrix $\mathbf{V}_{k}$ at the FD node $k$ :

$$
\begin{aligned}
\mathcal{O P} 2: \max _{\mathbf{C}_{k}, \mathbf{V}_{k}} & \mathcal{R}_{\mathrm{DL}}\left(\mathbf{V}_{k}\right) \\
\text { s.t. } & (\mathrm{C} 1),(\mathrm{C} 2), \\
& \left.\|\left(\mathbf{H}_{k, k}+\mathbf{C}_{k}\right) \mathbf{V}_{k}\right]_{(j,:)} \|^{2} \leq \lambda_{\mathrm{A}} \forall j=1,2, \ldots, M_{k} .
\end{aligned}
$$

To solve the latter problem we adopt an alternating optimization approach. Specifically, supposing that a realization of the analog canceler satisfying (C2) is given, we seek the TX digital precoder maximizing the downlink rate, while meeting $(\mathrm{C} 1)$ and the threshold $\lambda_{\mathrm{A}}$. Note that each realization of the analog canceler corresponds to a distinct MUX/DEMUX configuration. Let us assume that for $N$ taps (or $N$ AUX TXs, depending on the underlying canceler architecture) there are in total $L$ distinct realizations for the analog canceler, where $\mathbf{C}_{k}^{(\ell)}$ with $\ell=1,2, \ldots, L$ denotes the $\ell$-th canceler realization. Recall that $N$, which denotes the number of taps or AUX TXs, is decided offline upon hardware design as a function of size constraints, cost per tap and cost per AUX TX RF chain, or other hardware constraints. Examples of realizations for the analog canceler are given at the end of this section. We use the notation $\mathbf{V}_{k}^{(\ell)}$ to represent the precoder design solving $\mathcal{O} \mathcal{P} 2$ for each specific $\mathbf{C}_{k}^{(\ell)}$. The alternating optimization approach is repeated for $\mathbf{C}_{k}^{(\ell)} \forall \ell$ in order to find the best pair of canceler and precoder solving $\mathcal{O P} 2$. The solution for $\mathbf{V}_{k}^{(\ell)}$ given $\mathbf{C}_{k}^{(\ell)}$ is summarized in Algorithm 1. The precoder is iteratively constructed as the cascade ma$\operatorname{trix} \mathbf{F}_{k} \mathbf{G}_{k}$ with $\mathbf{F}_{k} \in \mathbb{C}^{N_{k} \times \alpha}$ and $\mathbf{G}_{k} \in \mathbb{C}^{\alpha \times d_{k}}$, where $\alpha$ is a positive integer taking the values $1 \leq \alpha \leq \alpha_{\max }$ and holds that $d_{k} \leq \min \left\{M_{q}, \alpha\right\}$. In general, $\alpha_{\max }=N_{k}$, however, for large transmission powers and strictly small values for $\lambda_{\mathrm{A}}$ it is advisable to set $\alpha_{\max }=\min \left\{M_{q}, N_{k}\right\}$. For each value of $\alpha$ we adopt a similar approach to [7] for the precoding design. Particularly, its $\mathbf{F}_{k}$ component aims at minimizing the impact of the residual SI MIMO channel $\widetilde{\mathbf{H}}_{k, k}$, whereas the goal of the $\mathbf{G}_{k}$ component is to maximize the rate of the effective downlink channel $\mathbf{H}_{q, k} \mathbf{F}_{k} \in \mathbb{C}^{M_{q} \times \alpha}$. Intuitively, parameter $\alpha$ represents the effective number of TX antennas after squeezing SI in the $N_{k}-\alpha$ least dominant modes of $\widetilde{\mathbf{H}}_{k, k}$ via the efficient use of $\mathbf{F}_{k}$. For the cases where $\mathbf{H}_{q, k} \mathbf{F}_{k}$ is a MIMO channel, the precoder $\mathbf{G}_{k}$ in Step 5 of Algorithm 1 is given by the open-loop or closed-loop precoding for this channel derived using [28], depending on whether $\mathbf{H}_{q, k}$ is unknown or known, respectively, at the transmit side of node $k$. In the simulation results shown later in Section 6 we will use open-loop precoding. When $M_{q}=1$ and $\alpha \geq 2, \mathbf{H}_{q, k} \mathbf{F}_{k}$ is a Multiple Input Single Output (MISO) channel, and if its knowledge is available at node $k$, the optimum precoding is Maximal Ratio Transmission (MRT). If $\mathbf{H}_{q, k} \mathbf{F}_{k}$ is a Single Input Multiple Output (SIMO) (i.e., for $M_{q} \geq 2$ and $\alpha=1$ ) or a scalar (i.e., for $M_{q}=\alpha=1$ ) channel, $\mathbf{G}_{k}$ is a scalar set to $\mathrm{P}_{k}^{1 / 2}$.

As seen from Step 15 of Algorithm 1 , the $\mathbf{V}_{k}^{(\ell)}$ solving $\mathcal{O P} 2$ for a specific $\mathbf{C}_{k}^{(\ell)}$ is given by $\mathbf{V}_{k, 1}^{(\ell)}$. This notation represents the precoder corresponding to the largest value of $\alpha$ that results in meeting constraint $\lambda_{\mathrm{A}}$; recall that $\alpha$ determines $\mathbf{F}_{k}$ and $\mathbf{G}_{k}$ dimensions. We denote the maximum value of $\alpha$ for the $\mathbf{C}_{k}^{(\ell)}$ design as $\alpha_{\ell}^{*}$, and also use the notation $\mathbf{V}_{k, m}^{(\ell)}$ with $m=1,2, \ldots, \alpha_{\ell}^{*}$ for the $m$-th candidate precoder solution for $\mathcal{O} \mathcal{P} 2$ given $\mathbf{C}_{k}^{(\ell)}$. Although, the included iterations for solving this problem could be terminated when $\mathbf{V}_{k, 1}^{(\ell)}$ is found, Algorithm 1 computes $\mathbf{V}_{k, m}^{(\ell)} \forall m$ meeting $\mathcal{O} \mathcal{P} 2$ 's threshold $\lambda_{\mathrm{A}}$ and optimizing the downlink rate for a given $\mathbf{C}_{k}^{(\ell)}$. Among those designs, the ones corresponding to lower values of $\alpha$ (i.e., those with increasing index $m$ ) naturally result in larger SI mitigation. Although this behavior is desirable for maximizing the uplink rate, $\mathbf{V}_{k, m}^{(\ell)}$ 's with larger $m$ (i.e., obtained from lower $\alpha$ ) yield lower downlink rates. On the contrary, $\mathbf{V}_{k, 1}^{(\ell)}$ maximizing the downlink rate creates the stronger SI signal contam- 


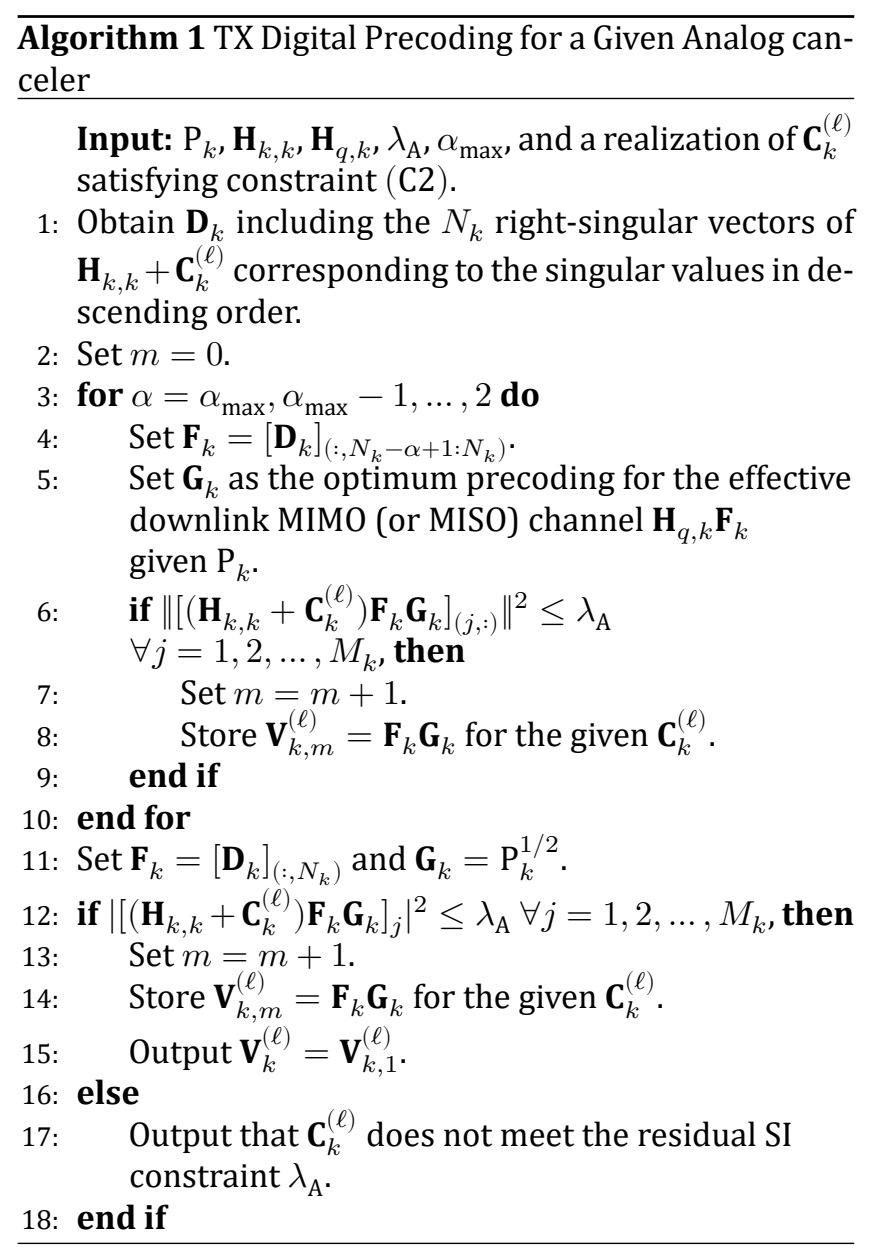

inating the uplink. Hence, our goal with Algorithm 1 is to capture this trade-off and obtain $\mathbf{V}_{k, m}^{(\ell)} \forall m$ solving $\mathcal{O P} 2$ for a given $\mathbf{C}_{k}^{(\ell)}$. Running this algorithm for all $L$ possible canceler realizations finally results in the joint canceler and precoder designs $\mathbf{C}_{k}^{(\ell)}$ and $\mathbf{V}_{k, m}^{(\ell)} \forall \ell=1,2, \ldots, L$ and $\forall m=1,2, \ldots, \alpha_{\ell}^{*}$, which are feasible candidate solutions for $\mathcal{O P} 2$. Those pairs will be used in Section 5.2 for obtaining the joint analog canceler and the TX/RX digital BF solution of $\mathcal{O P} 1$.

Algorithm 1 is executed at the FD MIMO node $k$ and has as inputs the MIMO channels $\mathbf{H}_{k, k}$ and $\mathbf{H}_{q, k}$, as well as a realization $\mathbf{C}_{k}^{(\ell)}$. Both $\mathbf{H}_{k, k}$ and $\mathbf{H}_{q, k}$ can be estimated through appropriately designed training processes at nodes $k$ and $q$, respectively. The latter matrix estimation can be fed back or not to node $k$ depending on whether open-loop or closed-loop MIMO operation, respectively, is adopted. We next discuss meaningful $\mathbf{C}_{k}^{(\ell)}$ realizations for both the proposed analog SI canceler architectures that provide insights on the effects of the $\mathbf{C}_{k}^{(\ell)}$ choice. Note that one can also consider reducing the search of canceler realizations in $\mathcal{O P} 2$ to a realization that is a deterministic function of $\mathbf{H}_{k, k}$ or to a desired subset of possible realizations.

Realizations $\mathbf{C}_{k}^{(\ell)}$ for the Multi-Tap canceler. For a given number of taps $N$ there are in total $\left({ }^{M_{k} N_{k}}{ }_{N}\right)$ ways to connect the taps from the available $N_{k}$ TX antennas to the available $M_{k} \mathrm{RX}$ antennas. This results in at most $L=$
$\left(\begin{array}{c}M_{k} N_{k} \\ N\end{array}\right)$ possible realizations for the multi-tap canceler. Each of those refers to a different $\mathbf{C}_{k}^{(\ell)}$ matrix and corresponds to a specific placement of the $N$ tap values inside $\mathbf{C}_{k}^{(\ell)}$; its remaining elements (i.e., $M_{k} N_{k}-N$ ) need to be set to zeros. One reasonable $\mathbf{C}_{k}^{(\ell)}$ intended for satisfying the SI constraint in $\mathcal{O P} 2$ is to obtain $\mathbf{L}_{1}, \mathbf{L}_{2}$, and $\mathbf{L}_{3}$ such that the resulting analog canceler matrix $\mathbf{C}_{k}^{(\ell)}$ has the $N$ tap values at the same elements with the $N$ largest in amplitude elements of $\mathbf{H}_{k, k}$. This $\mathbf{C}_{k}^{(\ell)}$ will result in cancelling the largest SI signal components. For example, suppose that $N_{k}=3, M_{k}=4$, and $N=2$ and that $\left[\mathbf{H}_{k, k}\right]_{2,1}$ and $\left[\mathbf{H}_{k, k}\right]_{4,2}$ are the two largest in amplitude elements of $\mathbf{H}_{k, k}$. In this case, we may design $\mathbf{L}_{1}, \mathbf{L}_{2}$, and $\mathbf{L}_{3}$ matrices as: $\mathbf{L}_{2}=\operatorname{diag}\left\{\left[\left[\mathbf{H}_{k, k}\right]_{2,1}\left[\mathbf{H}_{k, k}\right]_{4,2}\right]\right\},\left[\mathbf{L}_{1}\right]_{1,1}=\left[\mathbf{L}_{1}\right]_{2,2}=1$, and $\left[\mathbf{L}_{3}\right]_{2,1}=\left[\mathbf{L}_{3}\right]_{4,2}=1$. Other reasonable $\mathbf{C}_{k}^{(\ell)}$,s include the orderly column-by-column and row-by-row placement of the available $N$ tap values starting with the columns and rows, respectively, of $\mathbf{H}_{k, k}$ having the largest Euclidean norms. For example, suppose that $N_{k}=3, M_{k}=4$, $N=3$, and that the second RX antenna is the one most affected by SI (i.e., the one affected by the largest SI energy). Then, having the three tap values placed at the second row of $\mathbf{C}_{k}^{(\ell)}$ will focus on reducing the SI received at the second RX antenna element. Generally, having tap values placed at the $i$-th row results in reducing SI at the $i$-th RX antenna. In the simulation results with this architecture we opt for the latter canceler design, namely the row-by-row placement of the $N$ tap values, starting with $\mathbf{H}_{k, k}$ 's row having the largest Euclidean norm and continuing with the rest rows in descending ordering of Euclidean norms, if there are more taps to be assigned.

Realizations $\mathbf{C}_{k}^{(\ell)}$ for the Multi-AUX-TX canceler. To satisfy the constraint of $N$ AUX TXs, each canceler matrix needs to have $M_{k}-N$ all-zero rows. The $N$ nonzero rows specify the connection of the DEMUXs and the linear operation applied by $\mathbf{L}_{4}$. There are in total $\left(\begin{array}{c}M_{k} \\ N\end{array}\right)$ ways to connect the output of the $N$ AUX TXs to the $M_{k}$ RX antennas, and each way corresponds to a specific placement of the non-zero rows inside the canceler matrix. This results in at most $L=\left(\begin{array}{c}M_{k} \\ N\end{array}\right)$ possible realizations for the multi-AUX-TX canceler. One reasonable $\mathbf{C}_{k}^{(\ell)}$ realization, which we use in our simulation results for this architecture, corresponds to the case where the AUX TX RF chains are connected to the antennas that are receiving the largest SI energy. This realization targets $\mathbf{H}_{k, k}$ 's rows having the largest Euclidean norms. Connecting the $i$-th AUX TX RF chain to the $j$-th RX antenna corresponds to setting $\left[\mathbf{L}_{5}\right]_{j, i}=1$.

\subsection{Joint design of $\mathbf{C}_{k}, \mathbf{V}_{k}$, and $\mathbf{U}_{k}$}

Using the candidate designs $\mathbf{C}_{k}^{(\ell)}$ and $\mathbf{V}_{k, m}^{(\ell)} \forall \ell=1,2, \ldots, L$ and $\forall m=1,2, \ldots, \alpha_{\ell}^{*}$ for solving $\mathcal{O} \mathcal{P} 2$ from the approach in Section 5.1, we now proceed to the final joint design of the analog canceler and TX/RX digital BF at node $k$ maximizing the instantaneous FD rate. In particular, we formulate the following optimization problem using (12) and 
(13) for the computation of the best pair of $\mathbf{C}_{k}^{(\ell)}$ and $\mathbf{V}_{k, m}^{(\ell)}$ together with the optimum $\mathbf{U}_{k}$ :

$$
\begin{aligned}
\mathcal{O P} 3: \max _{\mathbf{U}_{k},\left\{\mathbf{C}_{k}^{(\ell)},\left\{\mathbf{V}_{k, m}^{(\ell)}\right\}_{m=1}^{\alpha_{\ell}^{*}}\right\}_{\ell=1}^{L}} & \mathcal{R}_{\mathrm{DL}}\left(\mathbf{V}_{k, m}^{(\ell)}\right) \\
& +\mathcal{R}_{\mathrm{UL}}\left(\mathbf{C}_{k}^{(\ell)}, \mathbf{V}_{k, m}^{(\ell)}, \mathbf{U}_{k}\right) \\
\text { s.t. } \quad & \left\|\left[\mathbf{U}_{k}\right]_{(i,:)}\right\|^{2}=1 \forall i=1,2, \ldots, d_{m} .
\end{aligned}
$$

To solve $\mathcal{O P} 3$ we adopt the following exhaustive search approach. For each of the $\sum_{\ell=1}^{L} \alpha_{\ell}^{*}$ pairs of analog canceler and TX digital precoder obtained in the previous step as candidate designs for solving $\mathcal{O} \mathcal{P} 2$, we compute $\mathbf{U}_{k}$ maximizing the uplink rate given by (13), while meeting its respective constraint included in both $\mathcal{O P} 1$ and $\mathcal{O P} 3$. Then, for each computed $\mathbf{U}_{k}$ and its corresponding $\mathbf{C}_{k}^{(\ell)}$ and $\mathbf{V}_{k, m}^{(\ell)}$ pair we calculate the achievable FD rate. The joint design maximizing the FD rate provides the solution for $\mathcal{O P} 3$. To solve the uplink rate maximization problem we assume that $\mathbf{H}_{k, m}$ and $\widetilde{\mathbf{H}}_{k, k}$ appearing in (13) and (14) are available at node $k$ through appropriately designed training phases. With the availability of this channel knowledge and a pair of $\mathbf{C}_{k}^{(\ell)}$ and $\mathbf{V}_{k, m}^{(\ell)}$, it can be shown that the $\mathbf{U}_{k}$ maximizing the UL rate is given using [29, Section 4.2] by $\mathbf{U}_{k}=\boldsymbol{\Gamma} \mathbf{W}^{\mathrm{H}}$, where $\mathbf{W} \in \mathbb{C}^{M_{k} \times d_{m}}$ has as columns the $d_{m}$ left singular vectors of $\boldsymbol{\Lambda}_{k}^{-1 / 2} \mathbf{E}_{k}^{\mathrm{H}} \mathbf{H}_{k, m}$ corresponding to its respective non-zero singular values. The diagonal matrix $\boldsymbol{\Lambda}_{k} \in \mathbb{C}^{M_{k} \times M_{k}}$ and the matrix $\mathbf{E}_{k} \in \mathbb{C}^{M_{k} \times M_{k}}$ are obtained from the eigenvalue decomposition of the interference-plus-noise covariance matrix $\mathbf{B}_{k} \in \mathbb{C}^{M_{k} \times M_{k}}$ at node $k$, which is defined as follows:

$$
\mathbf{B}_{k} \triangleq\left(\mathbf{H}_{k, k}+\mathbf{C}_{k}^{(\ell)}\right) \mathbf{V}_{k, m}^{(\ell)}\left[\mathbf{V}_{k, m}^{(\ell)}\right]^{\mathrm{H}}\left(\mathbf{H}_{k, k}+\mathbf{C}_{k}^{(\ell)}\right)^{\mathrm{H}}+\sigma_{k}^{2} \mathbf{I}_{M_{k}} .
$$

The eigenvalues of $\mathbf{B}_{k}$ are included in the main diagonal of $\boldsymbol{\Lambda}_{k}$, while the columns of $\mathbf{E}_{k}$ include their corresponding eigenvectors. The diagonal matrix $\boldsymbol{\Gamma} \in \mathbb{R}^{d_{m} \times d_{m}}$ ensures the constraint $\left\|\left[\mathbf{U}_{k}\right]_{(i,:)}\right\|^{2}=1 \forall i=1,2, \ldots, d_{m}$ is met. The $i$-th entry of $\boldsymbol{\Gamma}$ is equal to $1 /\left\|\left[\mathbf{W}^{\mathrm{H}}\right]_{(i,:)}\right\|$. For the special case of $N_{m}=1$ [17], which consequently results in $d_{m}=1$, the solution combining vector $\mathbf{w}_{k} \triangleq \mathbf{W}_{k} \in \mathbb{C}^{1 \times M_{k}}$ simplifies to the eigenvector corresponding to the maximum eigenvalue of the matrix $\mathbf{A}_{k} \in \mathbb{C}^{M_{k} \times M_{k}}$ given by [30]

$$
\mathbf{A}_{k} \triangleq \mathrm{P}_{m} \mathbf{B}_{k}^{-1} \mathbf{h}_{k, m} \mathbf{h}_{k, m}^{\mathrm{H}},
$$

where we have used the notation $\mathbf{h}_{k, m} \triangleq \mathbf{H}_{k, m} \in \mathbb{C}^{M_{k} \times 1}$. We note that for the practical case of imperfect analog cancellation, significant gains with the considered RX digital combining are feasible only when the following condition holds: $M_{k}-d_{k} \geq d_{m}$.

\subsection{Remarks}

We next provide some subtleties of our example FD MIMO design and possible extensions. We note however that, even without the following extensions, our presented design outperforms the SotA solutions, as will be shown in Section 6 including our performance evaluation results.

Remark 1: The presented solutions of $\mathcal{O P} 3$ for the analog cancellation and TX/RX digital BF are functions of the MIMO channel matrices $\mathbf{H}_{k, k}, \mathbf{H}_{k, m}$, and $\mathbf{H}_{q, k}$. This implies that the update of the BF settings as well as the settings of the canceler (values for the taps or AUX TX RF chains as well as MUX/DEMUX configurations) depend on the coherence time of the involved wireless channels.

Remark 2: Solving $\mathcal{O} \mathcal{P} 2$ is feasible when there exists at least one pair of $\mathbf{C}_{k}^{(\ell)}$ and $\mathbf{V}_{k, m}^{(\ell)}$ meeting the $\lambda_{\mathrm{A}}$ constraint. When such a pair does not exist, uplink communications are impossible to take place simultaneously with the downlink one (i.e., FD communications for the given $N$ and $\lambda_{\mathrm{A}}$ is infeasible). We note that, for our FD rate results appearing in Section 6.4, we only focus on scenarios where solving $\mathcal{O P} 2$ is feasible. For cases where a $\mathbf{C}_{k}^{(\ell)}$ and $\mathbf{V}_{k, m}^{(\ell)}$ pair satisfying $\lambda_{\mathrm{A}}$ does not exist, $\mathcal{O P} 1$ can be solved via half duplex communications, and there is no need for a canceler design. In this case, the $\mathcal{O P} 1$ solution is either the precoder maximizing the downlink rate or the combiner maximizing the uplink one, depending on which of the two results in the maximum half duplex rate. If we relax the SI constraint in $\mathcal{O P} 1$ and $\mathcal{O P} 2$ to a subset, instead of all, $M_{k}$ RX RF chains (i.e., suppose that the constraint becomes $\left\|\left[\widetilde{\mathbf{H}}_{k, k} \mathbf{V}_{k}\right]_{(j,:)}\right\|^{2} \leq \lambda_{\mathrm{A}} \forall j=1,2, \ldots, M_{k}^{\prime}$ with $M_{k}^{\prime}<M_{k}$ ),FD communications are more probable to be feasible for a given $\mathrm{N}_{p}$ and $\lambda_{\mathrm{A}}$. This happens because with this relaxation we allow uplink communications even when there exists at most $M_{k}-M_{k}^{\prime} \mathrm{RX} \mathrm{RF}$ chains experiencing average residual SI power larger than $\lambda_{\mathrm{A}}$. However, those saturated RX RF chains should not be considered for reliable reception, hence, they should be deactivated for uplink communications via adequate antenna selection. Under this strategy, the uplink MIMO matrix is denoted by $\mathbf{H}_{k, m}^{\prime} \in \mathbb{C}^{M_{k}^{\prime} \times N_{m}}$ being a submatrix of $\mathbf{H}_{k, m}$, where the rows corresponding to the saturated RX $\mathrm{RF}$ chains have been excluded. It is finally noted that the value for $M_{k}^{\prime}$, and to which specific RX RF chains the $\lambda_{\mathrm{A}}$ constraint is imposed, will impact the achievable uplink rate, and hence, the feasible FD communications.

\section{SIMULATION RESULTS AND DISCUSSION}

The performance of the wireless communication scenario illustrated in Fig. 1 using the FD MIMO design presented in Section 5 is evaluated. In Section 6.1 we describe the benchmark approaches with which our solutions will be compared. The simulation parameters and assumptions are then detailed in Section 6.2, whereas the SI mitigation capability and achievable rate results for different hardware complexity levels are presented in Secs. 6.3 and 6.4. 


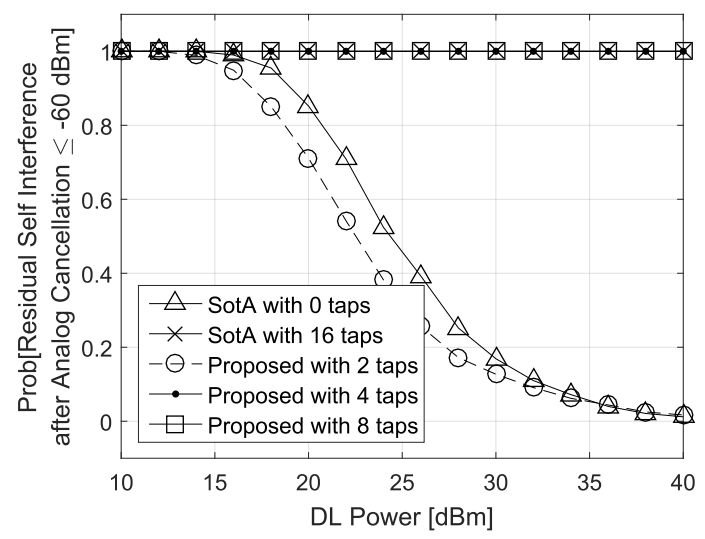

(a) Multi-tap canceler, $M_{q}=N_{m}=1$.

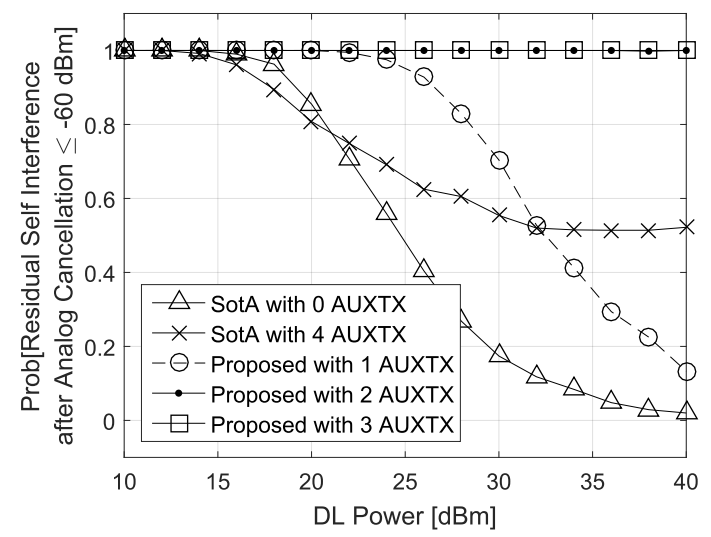

(c) Multi-AUX-TX canceler, $M_{q}=N_{m}=1$.

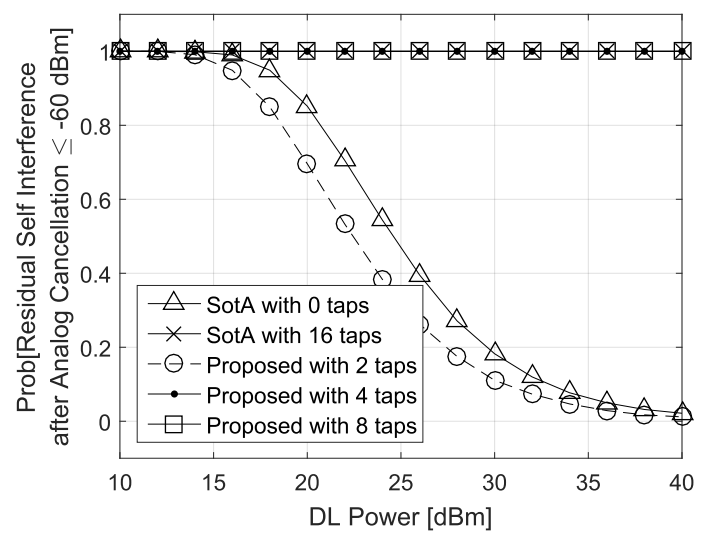

(b) Multi-tap canceler, $M_{q}=N_{m}=4$.

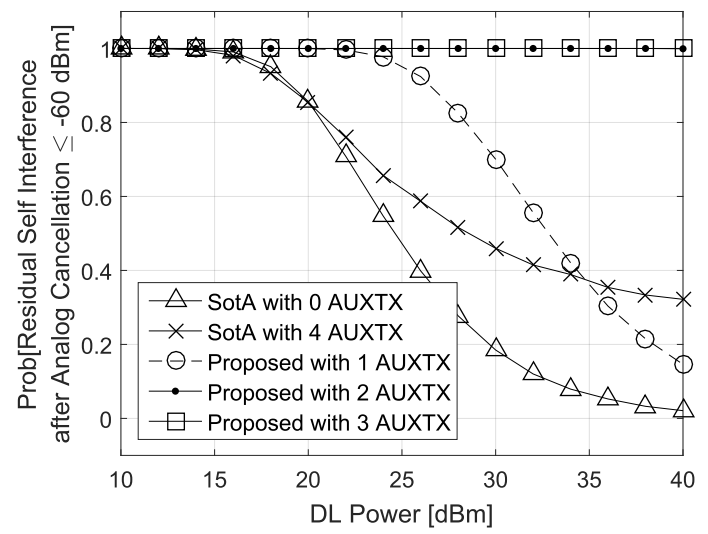

(d) Multi-AUX-TX canceler, $M_{q}=N_{m}=4$.

Fig. 4 - Probability of the residual SI power at each of the RX RF chains being less or equal to $\lambda_{\mathrm{A}}=-60 \mathrm{dBm}$ versus the DL TX power $\mathrm{P}_{k}$ for $M_{k}=N_{k}=4$.

\subsection{Compared FD MIMO designs}

We compare the presented FD MIMO designs versus the combined cancellation and spatial suppression design presented in [4] as well as the digital BF design proposed in [7]. We note that the designs presented in $[20,21]$ were not considered in the results that follow due to the fact that they are only applicable to UpLink (UL) and DownLink (DL) communications with $d_{k}=d_{m}=1$, whereas our proposed solutions hold for $d_{k}, d_{m} \geq 1$. A detailed description of the FD MIMO designs that will be compared is provided below.

Design 1: Proposed with $N$ taps. This is the presented FD MIMO design with an $N$-tap analog canceler. Compared with the SotA architectures $[6,15]$ requiring at least $M_{k} N_{k}$ taps, our canceler results in $100\left(1-N /\left(M_{k} N_{k}\right)\right) \%$ reduction in the required taps' numbers. The TX/RX digital BF as well as the settings for the canceler at the FD MIMO node $k$ are computed as presented in Section 5. For $\mathbf{H}_{q, k} \mathbf{F}_{k}$ being a MIMO channel, we have adopted open-loop MIMO precoding for the computation of $\mathbf{G}_{k}$.

Design 2: Proposed with N AUXTX. This is the presented FD MIMO design for the case of multi-AUX-TX canceler with $N$ AUX TX RF chains. Compared with the SotA architectures $[14,16]$ which require at least $M_{k}$ AUX TXs, our canceler results in $100\left(1-N / M_{k}\right) \%$ reduction in the required number of AUX TXs. We have again used Section 5 for the computation of TX/RX digital BF as well as the canceler settings at the FD MIMO node $k$. The $\mathbf{G}_{k}$ computation was the same as for Design 1.

Design 3: SotA with $M_{k} N_{k}$ taps. This refers to a combination of time domain analog cancellation with spatial suppression, as proposed in [4]. The TX beamformer is designed to minimize SI caused from this operation by using null space projection [4] for this communication side. The RX BF was proposed to be an MMSE filter in [4], we however utilize the optimum combiner $\mathbf{U}_{k}$ obtained using [29, Section 4.2], as explained in Section 5. Hence, we use the same combiner as in Designs 1 and 2. The time domain cancellation is a canceler that requires in total $M_{k} N_{k}$ taps (i.e., one tap per TX-RX RF chain), as in the SotA schemes $[6,15]$. We have made the same assumptions for the hardware capabilities of the taps for this design as in Design 1. Design 4: SotA with $M_{k}$ AUXTX. This design is similar to Design 3 but uses AUX TXs in place of the analog taps. It particularly combines time domain cancellation with spatial suppression [4]. The former is an analog canceler requiring a total of $M_{k}$ AUX TX RF chains (i.e., one AUX TX RF chain per RX RF chain), as in the SotA schemes $[14,16]$. In addition, the hardware capabilities of each AUX TX are 
considered the same with our Design 2. TX digital BF is designed for SI minimization from the TX side, whereas $\mathrm{RX}$ digital $\mathrm{BF}$ is given by $\mathbf{U}_{k}$, as described in Section 5 .

Design 5: SotA with 0 taps/0 AUXTX. This is the SoftNull method presented in [7] that does not adopt analog cancellation, relying solely on TX digital BF to reduce SI at the $\mathrm{RX}$ antennas of node $k$. Any residual SI is handled by the $\mathrm{RX}$ digital combiner. The combiner $\mathbf{U}_{k}$ used in the previous designs is used for the latter purpose.

\subsection{Simulation parameters}

We have assumed Rayleigh fading and a path loss of $110 \mathrm{~dB}$ for both the DL $\mathbf{H}_{q, k}$ and UL $\mathbf{H}_{k, m}$ channels. The SI channel $\mathbf{H}_{k, k}$ is assumed to be subject to Ricean fading with $\kappa$-factor equal to $35 \mathrm{~dB}$ and path loss of $40 \mathrm{~dB}$ [31]. All involved wireless channels are assumed to be Independent and Identically Distributed (IID), and perfectly estimated at the receivers (i.e., at the RXs of nodes $k$ and $q$ ). We have used 1000 independent channel realizations for all statistical results. The DL transmit power $\mathrm{P}_{k}$ was set between $10 \mathrm{dBm}$ and $40 \mathrm{dBm}$, and the UL transmit power $\mathrm{P}_{m}$ was set $20 \mathrm{~dB}$ lower, hence, spanning a range from $-10 \mathrm{dBm}$ to $20 \mathrm{dBm}$ [32]. The noise floor at node $q$ is $-90 \mathrm{dBm}$ and at node $k$ is $-110 \mathrm{dBm}$. The latter values are typical ones for small cell base stations and mobile terminals. Following the findings of [3], we consider a 14-bit ADC at node $k$ that renders digital SI mitigation of approximately $50 \mathrm{~dB}$ feasible. This means that for the noise floor of $-110 \mathrm{dBm}$ at node $k$ the residual SI after analog cancellation (i.e., at each RX RF chain's input) must be less than $-60 \mathrm{dBm}$. In Appendix A we present the two realistic models used for simulating non-ideal analog canceler hardware. The one model concerns the proposed multi-tap canceler architecture and the other the multi-AUX-TX one. According to these models, the multi-tap canceler is capable of delivering approximately $60 \mathrm{~dB}$ of analog cancellation per tap, whereas the multi-AUX-TX canceler offers approximately $35 \mathrm{~dB}$ of cancellation per AUX TX RF chain.

\subsection{Self-interference mitigation capability}

We consider a $4 \times 4$ FD MIMO node $k$ (i.e., $M_{k}=N_{k}=4$ ) and two different cases for the number of antennas at nodes $q$ and $m$ : the single-antenna case (i.e., $M_{q}=N_{m}=$ 1) and the multi-antenna with $M_{q}=N_{m}=4$. We investigate in Fig. 4 the probability that the residual SI after analog cancellation meets the constraint of being less than $\lambda_{\mathrm{A}}=-60 \mathrm{dBm}$. Results are shown for both proposed multi-tap and multi-AUX TX architectures for various hardware complexity levels, as implicated by different values of $N$ for the taps and AUX TXs, respectively. Within Fig. 4, we also sketch results for SotA designs with $N=16$ taps and with $N=4$ AUX TXs, as well as for the only digital SotA solution (i.e., 0 taps or 0 AUX TXs). For the latter design, we have one DL stream for the precoder, since this was the configuration yielding the largest SI reduction, however, as shown from all subfigures of Fig. 4, for $\mathrm{P}_{k} \geq 15 \mathrm{dBm}$, this design is incapable of guaranteeing residual SI power levels at any of the RX RF chains below the required $\lambda_{\mathrm{A}}=-60 \mathrm{dBm}$. Figs. $4 \mathrm{a}$ and $4 \mathrm{~b}$ demonstrate that the proposed multi-tap-based design ensures that the residual SI power satisfies the $\lambda_{\mathrm{A}}$ constraint for all considered TX powers for $N=4$ and $N=8$ taps, which translates to $25 \%$ and $50 \%$ less taps compared to the SotA requiring $N=16$ taps. In addition, Figs. $4 \mathrm{c}$ and $4 \mathrm{~d}$ showcase that the proposed multi-AUX TX solution with $N=2$ and $N=3$ AUX TXs is the only one based on AUX TXs that is capable of offering residual SI power below $-60 \mathrm{dBm}$ for all $\mathrm{P}_{k}$ values. Actually, the SotA design with $N=4$ AUX TXs (i.e., with $50 \%$ and $25 \%$ more AUX TXs than the $N=2$ and $N=3$ AUX TXs cases) cannot meet the residual constraint for $\mathrm{P}_{k} \geq 15 \mathrm{dBm}$.

We now investigate in more detail how our proposed joint analog cancellation and BF design adapts in order to meet the constraint on residual SI, while providing spatial resources for DL and UL communications. Recall that $\alpha$ used in the precoder solving $\mathcal{O} \mathcal{P} 3$ determines the effective number of TX antennas used for DL data transmission. An $\alpha$ close to $N_{k}$ means that the TX BF of the FD node is using more antenna resources for improving DL than for SI reduction. Therefore, $\alpha$ determines the trade-off between acceptable SI levels as well as DL and UL achievable rates. In Figs. 5a and 5b, we illustrate the average values of $\alpha$ chosen by our FD MIMO design as functions of the DL and UL TX powers for the case of the multi-tap architecture (for $N=4$ and $N=8$ taps) and multi-AUX TX architecture (for $N=2$ and $N=3$ AUX TXs), respectively, and for $M_{q}=N_{m}=1$ and $M_{q}=N_{m}=4$. From these figures we observe that for a given $M_{q}$ and $N_{m}$, the value of $\alpha$ increases as the number of taps (or AUX TXs) increases. For example, in Fig. 5a for $M_{q}=N_{m}=1$, the values of $\alpha$ for $N=8$ taps are always larger than the values of $\alpha$ for $N=4$ taps. The more the taps (or AUX TXs), the more analog canceler resources are provided for SI mitigation, and hence, less antenna resources are required for this mitigation in order to meet the residual SI constraint. This is why our algorithm chooses a larger $\alpha$ as the number of taps (or AUX TXs) increases. Thus, the results in Fig. 5 verify that our FD MIMO design is capable of judiciously dividing the burden of SI mitigation between the analog canceler and the TX digital BF by taking into account the resources available for analog cancellation.

Another observation from the results in Fig. 5 is that as the number $M_{q}$ of RX antennas in DL and/or the number $N_{m}$ of TX antennas in UL increase, our FD MIMO design tends to be more conservative in the choice of $\alpha$ since it chooses a smaller value for $\alpha$. For example, in Fig. 5 a for the case of $N=8$ taps, the values of $\alpha$ for $M_{q}=N_{m}=1$ are larger than those for $M_{q}=N_{m}=4$. The reason for this behavior is as follows. Recall that the number of UL streams is equal to $d_{m} \leq \min \left\{M_{k}, N_{m}\right\}$. Since $M_{k}=4$, then as $N_{m}$ increases from 1 to 4 , there will be more streams in the UL communications. This increment of UL streams makes the design of TX digital BF more demanding, since it has to steer SI away from these several incoming UL streams 


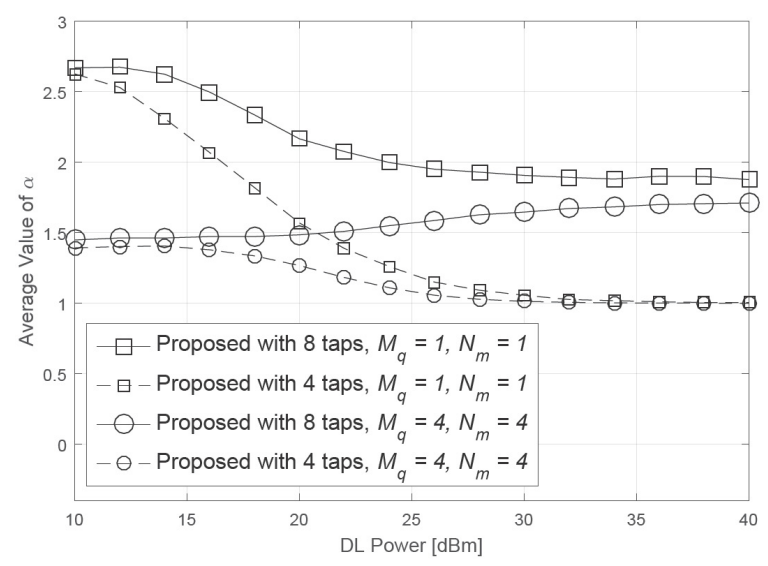

(a) Multi-tap architecture.

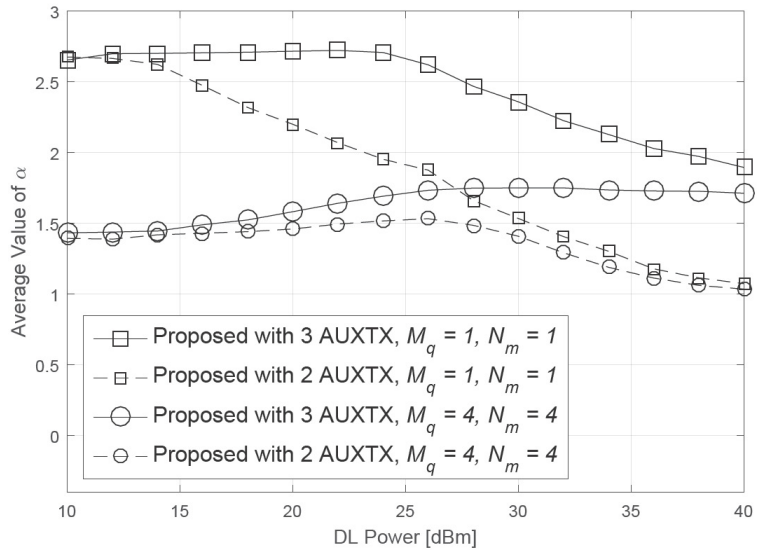

(b) Multi-AUX TX architecture.

Fig. 5 - Average $\alpha$ value for the proposed FD MIMO design versus DL TX power $P_{k}$ and for UL TX power $P_{m}=P_{k}[\mathrm{dBm}]-20[\mathrm{~dB}]$ for $M_{k}=N_{k}=4$.

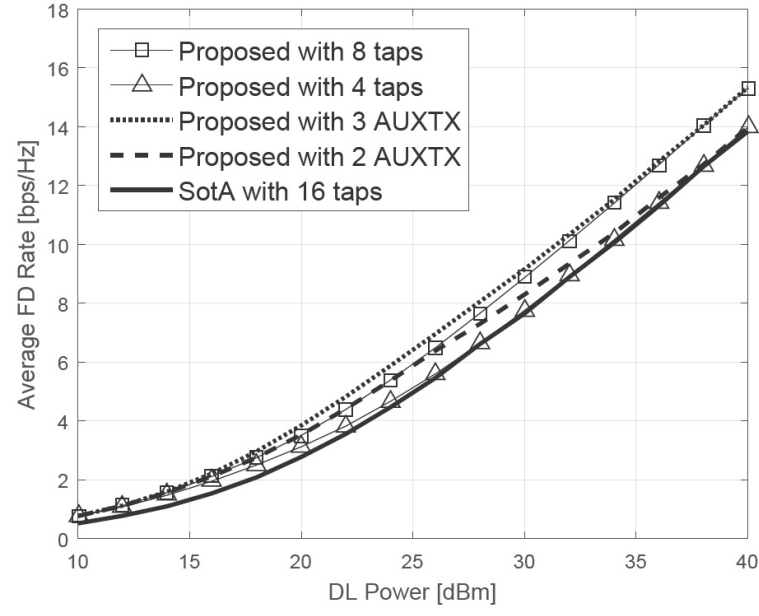

(a) $M_{q}=N_{m}=1$.

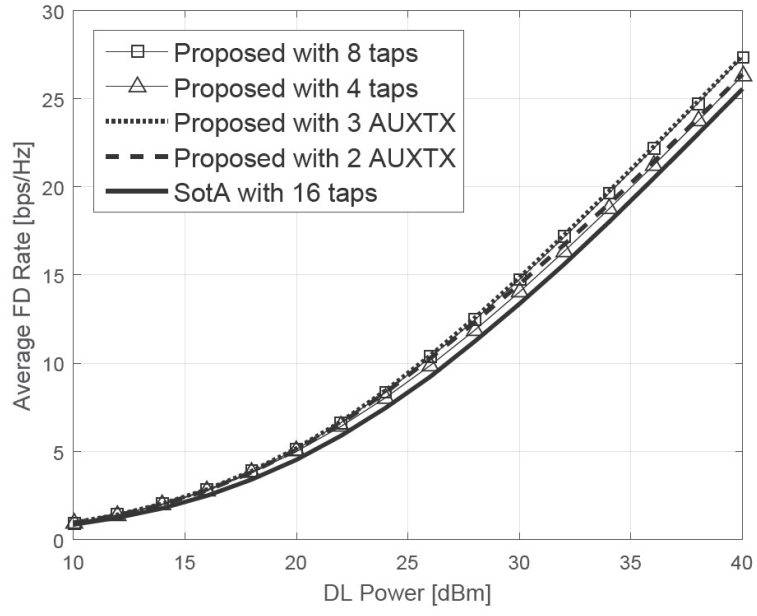

(b) $M_{q}=N_{m}=4$.

Fig. 6 - Average FD rates versus DL TX power $P_{k}$ and for UL TX power $P_{m}=P_{k}[\mathrm{dBm}]-20[\mathrm{~dB}]$ for $M_{k}=N_{k}=4$.

in order to maximize the FD rate. Thus, our FD MIMO design chooses the small $\alpha=1$ so that the FD node can put more effort into SI mitigation. Serving less streams in DL, by choosing a lower $\alpha$, allows to devote more TX spatial directions at the FD node $k$ for SI mitigation. This showcases the reconfigurability of the TX digital BF design to satisfy the FD rate maximization objective, while meeting hardware and SI constraints.

\subsection{Achievable rates}

We plot the ergodic DL, UL, and FD rates in $\mathrm{bps} / \mathrm{Hz}$ as functions of the TX powers for the FD MIMO systems considered in Figs. 4 and 5, using the algorithmic designs satisfying the constraint of having residual SI power level after analog cancellation lower than $\lambda_{\mathrm{A}}=-60 \mathrm{dBm}$. We do the same for the SotA algorithm with $N=16$ taps, which is the only considered design from the previous art meeting the latter SI constraint. Starting with Fig. 6, the FD rate performance of the proposed multi-tap and multi-AUX TX designs with $N=\{4,8\}$ taps and $N=\{2,3\}$ AUX TXs, respectively, is illustrated in comparison with the SotA design having $N=16$ taps. As seen from both Figs. 6a and 6b with $M_{q}=N_{m}=1$ and $M_{q}=N_{m}=4$, respectively, the multi-tap design with $N=4$ and $N=8$ taps (i.e., $75 \%$ and less $50 \%$ less taps than SotA) yields similar or better performance to SotA. Fig. 6 also showcases the superiority of the proposed multi-AUX TX design with respect to the SotA one having $N=16$ taps.

In Fig. 7 we focus on the achievable DL and UL rates with the proposed multi-tap and multi-AUX TX designs with $N=8$ taps and $N=3$ AUX TXs, respectively, and with the SotA design with $N=16$ taps. It is shown that our proposed TX digital precoder results in a larger DL rate for $M_{q}=N_{m}=\{1,4\}$. The same trend holds for the UL rate with the proposed joint design of analog cancellation and RX digital combining. This behavior witnesses the reconfigurability of the proposed joint design, which results in both larger UL and DL gains compared to SotA, as the number of antennas at nodes $q$ and $m$ increase. 

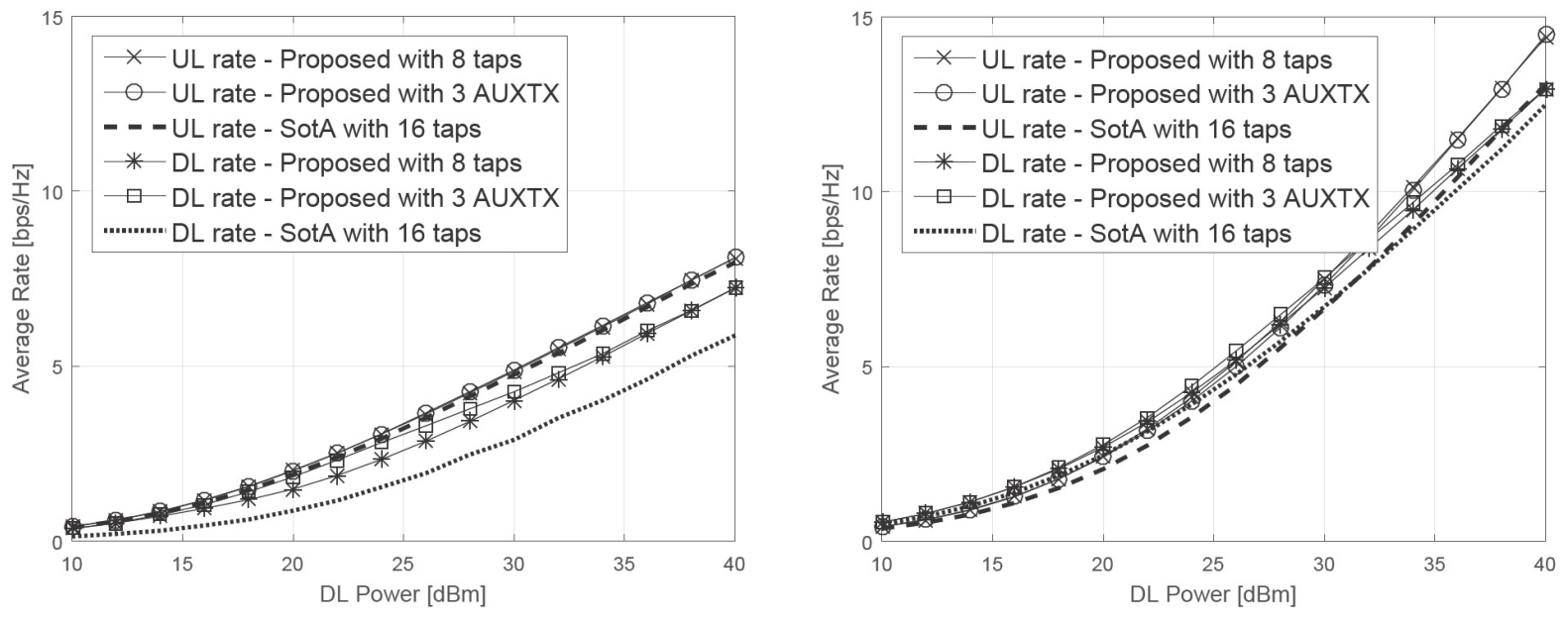

(a) $M_{q}=N_{m}=1$.

(b) $M_{q}=N_{m}=4$.

Fig. 7 - Average rates versus DL TX power $P_{k}$ and for UL TX power $P_{m}=P_{k}[\mathrm{dBm}]-20[\mathrm{~dB}]$ for $M_{k}=N_{k}=4$.

\section{RECENT FD MIMO APPLICATIONS AND FUTURE DIRECTIONS}

In this section, we briefly introduce recent indicative STAR schemes leveraging the presented FD MIMO hardware architecture with multi-tap cancellation, and present open challenges and research directions for future FD MIMO communication systems.

\subsection{STAR applications}

The performance of the presented framework in the presence of hardware imperfections, TX IQ imbalance, nonlinear power amplification, and imperfect Channel State Information (CSI) was recently investigated in [25, 34]. In particular, aiming at suppressing the residual linear and nonlinear SI signal below the noise floor, [25] presented a digital SI cancellation technique that was jointly designed with the configuration of the analog taps and digital BF. In [34], the authors focused on two-way FD MIMO systems and designed BF schemes, considering models for imperfect hardware and CSI errors. Very recently in [23], a multi-tap wideband analog SI canceler architecture, whose number of taps does not scale either with the number of transceiver antennas or the number of multipath SI components, was designed for wideband FD MIMO communication systems with Orthogonal Frequency-Division Multiplexing (OFDM). Therein, an adaptive digital SI cancellation technique was presented that reduces the residual SI signal estimation parameters. The presented FD MIMO architecture with multi-tap cancellation was recently extended in [35] for transceivers capable of realizing hybrid A/D BF [33]. The proposed analog SI canceler interconnects a subset of the outputs of the TX RF chains to a subset of the inputs to the RX RF chains in order to ensure that the residual SI signal after A/D TX precoding and analog RX combining remains below the noise floor. Very recently, in $[36,37,38]$, it was shown that the number of analog taps can be scaled to the product of the number of transmit beams and the number of RX RF chains, and the interplay between digital BF and analog cancellation for improving the design degree of freedoms was thoroughly investigated.

Capitalizing on the presented reduced-complexity STAR architectures, a transmission scheme exploiting channel reciprocity for joint downlink beamformed information data communications and uplink channel estimation, through training data transmission, was presented initially for a two-way FD MIMO system in [39] and then for multiple users in [40]. Very recently, the authors in [41] leveraged the FD massive MIMO framework with hybrid A/D BF of [35] and presented a direction-assisted beam management scheme for millimeter Wave (mmWave communications. In this scheme, UL direction-of-arrival estimation and DL analog BF were realized simultaneously, aiming at reducing the control information exchange overhead that impacts spectral efficiency.

\subsection{Open challenges and future directions}

The performance of the presented architectures has been investigated for ideal selection networks in the analog SI canceler, i.e., for ideal MUXs/DEMUXs of arbitrary values $N$ for the analog cancellation units. Additionally, the available operation algorithms are suboptimal requiring an offline investigation for the adequate value for $N$. Therefore, further studies are needed considering selection networks with practical losses and feasible $N$ numbers for the MUXs/DEMUXs sizes, as well as realistic correlated channels for planar antenna arrays. Additionally, the insertion loss induced in practice by each analog tap needs to be accurately characterized, since this will determine the feasible upper bound in the number of taps comprising the analog canceler. Furthermore, optimized algorithms with low computational complexity for multiuser and/or multi-cell simultaneous UL and DL operations need to be derived. To this end, the FD operation, which inevitably contributes to the overall network 
interference, needs to be efficiently incorporated and handled into the next generation Non-Orthogonal Multiple Access (NOMA) techniques. The design of analog and digital TX/RX beam codebooks for SI mitigation is another important research topic towards the practical deployment of FD MIMO transceivers. We next detail some of the most promising future directions, and their challenges, for FD MIMO communication systems.

mmWave and THz communications: Given the limited spectral resources at frequencies below $6 \mathrm{GHz}$, the $5 \mathrm{G} \mathrm{New}$ Radio has adopted mmWave frequencies to accommodate high data rate communications, and 6G is expected to support wireless links at subTHz [1]. To compensate for the high path loss at those high frequency bands, highly directive $A / D B F$ is necessary. Hence, combining FD MIMO radios with mmWave and $\mathrm{THz}$ technologies is a promising approach to reach higher spectral efficiencies. Interestingly, the STAR applications of the recent FD MIMO framework $[39,40]$ can be used for low latency analog beam tracking, paving the way for beyond $5 \mathrm{G}$ standalone mmWave communications. For example, the beam refinement for multiple users can take place in the UL simultaneously with their DL data communications from the FD MIMO base station. However, the nonlinearities of the power amplifiers become the bottleneck of SI cancellation in mmWave and THz sfrequencies. The RF imperfections will not be sufficiently suppressed in the analog domain with reduced hardware complexity, and sophisticated digital SI cancellation will be needed possibly with the aid of digital RX BF.

Integrated communications and sensing: The STAR applications $[39,40]$ constitute only a portion of the potential of the FD MIMO technology for integrated communications and sensing applications. In fact, the FD operation can enable joint communications and radar [42, 43], offering re-usability of the available resources and integration of advanced sensing capabilities in future wireless systems. To this end, the recent extension [35] of the presented FD MIMO framework can be exploited for realizing highly flexible multiple beams for both communications and radar, trading off the complexity of SI cancellation with that for the sensing resolution. Furthermore, it is interesting to consider the presented reducedcomplexity architectures and schemes for integrated access and backhaul, which is of particular interest for 5G and beyond wireless systems [2].

Channel estimation schemes: Most FD MIMO techniques rely on the idealized simplifying assumption of the availability of perfect CSI; however, this assumption is unrealistic. Indeed, the CSI acquired at the FD nodes is imperfect due to estimation errors induced by: $i$ ) nonlinear hardware components; ii) phase noise from the device's oscillators; and iii) channel aging caused by the mobility of the users. Those CSI errors will be exacerbated at the FD node when massive numbers of TX and RX antennas are used. In addition, as shown in [25], it is very hard to completely cancel the SI signal in the practical case of imperfect CSI. Hence, CSI estimation is a particularly criti- cal issue in FD MIMO systems. The same holds for channel aging, which requires sophisticated channel tracking algorithms. To maximize the ergodic mutual information, which is a non-convex function of the power allocation, efficient techniques for optimizing the power/pilotoverhead allocation vectors need to be developed.

Machine-learning-based configurations: Artificial Neural Networks (ANNs) are lately gaining substantial attention in wireless communications [44] as an efficient means to deal with hardware nonlinearities and to tame wireless channel dynamics $[45,46]$. It is appealing to consider efficient hardware implementations of ANNs to handle the nonlinearities of the multiple power amplifiers in the FD MIMO architectures, especially when considered for high-frequency communications. This will enable more efficient analog SI cancelers, thus, increasing the flexibility of A/D TX/RX BF. In addition, supervised and reinforcement learning techniques can be deployed for traffic predictions in order to enable dynamic scheduling of UL and DL users in multiple interfering FD massive MIMO connections. With such approaches, the large overhead of multiple pilot-assisted massive MIMO channel estimates will be avoided.

Massive metasurface antennas: Incorporating reconfigurable metasurfaces in wireless networks has been recently advocated as a revolutionary means to transform any naturally passive wireless propagation environment into a dynamically programmable one $[47,48,49]$. This can be accomplished by deploying cost-effective and easy to coat metasurfaces to the environment's objects, thus, offering increased environmental intelligence for the scope of diverse wireless networking objectives [50]. Metasurfaces are artificial planar structures of multiple reconfigurable radiating metamaterial elements that can be programmed to reflect an incoming electromagnetic field in a reconfigurable way (passive metasurfaces [51]), or can be used as TX or RX antenna arrays (hybrid [52] or active [53] metasurfaces). Interesting directions of research are the investigation of FD massive MIMO operation in networks empowered be passive metasurfaces, and the design of FD MIMO architectures with massive numbers of metasurface antennas. In the former direction, efficient NOMA techniques to manage FD-based reflections need to be devised, whereas in the latter, hybrid A/D TX/RX BF schemes will be required.

\section{CONCLUSIONS}

In this paper, we presented a detailed overview of two recent SI mitigation schemes for FD MIMO systems with reduced hardware complexity. Each presented scheme includes a reduced complexity analog canceler architecture, one based on analog taps and the other on AUX TXs. The main simplification of the multi-tap canceler hardware was obtained via the use of MUXs/DEMUXs for signal routing among the TX and RX RF chains and the reduced number of taps, as well as the joint design of the tap values and MUXs/DEMUXs configuration with the TX/RX 
digital BF filters. Similar simplification was gained by the multi-AUT-TX canceler hardware, where the reduced number of AUX TX RF chains were jointly designed with MUXs/DEMUXs and TX/RX digital BF. We have presented a general optimization framework for the joint design of analog SI cancellation and digital BF, and detailed a specific algorithmic solution targeting the FD rate maximization. The performance evaluation results, based on the proposed realistic models for non-ideal analog cancellation hardware, demonstrated that the presented designs can be implemented with less cancellation elements (less taps or AUX TXs) than SotA ones, while achieving larger FD rates. We discussed recent STAR schemes capitalizing on the overviewed hardware and algorithmic framework, and presented some open challenges and research directions for future FD MIMO communication systems and their promising wireless applications.

\section{A. SIMULATION MODELS FOR THE ANALOG CANCELER HARDWARE}

We next present two simulation models for non-ideal analog canceler hardware. The first model is considered for the presented multi-tap canceler architecture and the second for the multi-AUX-TX canceler architecture.

\section{A.1 Model for the analog taps}

In the ideal hardware case, the amplitude and phase of each of the analog taps take any desired arbitrary value. However, the settings for the attenuator and phase shifter comprising a tap take only discrete value steps when realistic hardware is considered. Consequently, we assume that each tap is set with steps of $0.02 \mathrm{~dB}$ for attenuation and of $0.13^{\circ}$ for phase; these values match the step values reported in [15]. Thus, for each analog tap in our simulations, the phase setting has a random phase error uniformly distributed between $-0.065^{\circ}$ and $0.065^{\circ}$, and the amplitude setting has a random amplitude error uniformly distributed between $-0.01 \mathrm{~dB}$ and $0.01 \mathrm{~dB}$. More specifically, in our simulations we do not use the ideal cancellation values given by $\mathbf{C}_{k}$, instead we use a more realistic noisy version given by $\hat{\mathbf{C}}_{k} \in \mathbb{C}^{M_{k} \times N_{k}}$. The $N$ nonzero elements of $\hat{\mathbf{C}}_{k}$ are the same $N$ non-zero elements of $\mathbf{C}_{k}$, but affected by a random phase and magnitude error. More specifically, for the $(i, j)$-th non-zero element of $\mathbf{C}$ due to the $n$-th analog tap, we compute its noisy version as follows:

$$
\left[\hat{\mathbf{C}}_{k}\right]_{i, j}=\left[\mathbf{C}_{k}\right]_{i, j} \exp \left(j \alpha_{n}\right) 10^{\beta_{n} / 20}
$$

for $i=1,2, \ldots, M_{k}$ and $j=1,2, \ldots, N_{k}$, where $\alpha_{n}$ is uniformly distributed over the interval $[-0.065 \pi / 180,0.065 \pi / 180]$ and $\beta_{n}$ is uniformly distributed over the interval $[-0.01,0.01]$. In the latter expression, $\alpha_{n}$ and $\beta_{n}$ represent the phase and magnitude errors, respectively, due to the non-ideal hardware at the $n$-th tap. We also assume that $\alpha_{n}$ and $\beta_{n} \forall n=1,2, \ldots, N$ are IID random variables. Applying analog cancellation with a tap exhibiting $0.065^{\circ}$ phase and $0.01 \mathrm{~dB}$ magnitude errors, respectively, results in approximately $60 \mathrm{~dB}$ of SI cancellation. Hence, the considered multi-tap canceler architecture in our simulations is capable of delivering approximately $60 \mathrm{~dB}$ of analog cancellation per tap.

\section{A.2 Model for the AUX TX RF chains}

One known characteristic of the canceler architecture based on AUX TX RF chains is that the SI signal used for cancellation at the RX side is obtained from the digital domain. Due to this fact, this cancellation signal does not include the inherit non-linearities of the actually transmitted SI signal; these non-linearities exist in real-world TX RF chain hardware. As has been described in [54], one such non-linearity is the oscillator phase noise at the TX RF chains and AUX TX RF chains. This non-linearity source has been shown to be a dominant bottleneck for the performance of analog cancelers based on AUX TX RF chains. Thus, our model includes phase noise effects.

Let us denote by $\phi_{i}^{A}$ and $\phi_{j}^{T}$ with $i=1,2, \ldots, N$ and $j=1,2, \ldots, N_{k}$ the phase noise due to the $i$-th AUX TX RF chain and $j$-th TX RF chain, respectively. We use the matrix notation $\boldsymbol{\Phi}_{k} \in \mathbb{C}^{N \times N_{k}}$ to represent the imperfections due to phase noise. Each $(i, j)$-th element of this matrix captures the phase noise mismatch between the $i$-th AUX TX RF chain and $j$-th TX RF chain, and is expressed as:

$$
\left[\Phi_{k}\right]_{i, j}=\exp \left(j \phi_{i}^{A}\right)-\exp \left(j \phi_{j}^{T}\right)+1
$$

for $i=1,2, \ldots, N$ and $j=1,2, \ldots, N_{k}$. In our simulations we do not use the ideal cancellation values given by matrix $\mathbf{C}_{k}$, we instead use a more realistic noisy version given by $\hat{\mathbf{C}}_{k}$, which is computed as $\hat{\mathbf{C}}_{k}=\mathbf{L}_{5} \hat{\mathbf{L}}_{4}$, where the matrix $\hat{\mathbf{L}}_{4} \in \mathbb{C}^{N \times N_{k}}$ is defined as $\hat{\mathbf{L}}_{4} \triangleq \boldsymbol{\Phi}_{k} \odot \mathbf{L}_{4}$. Notice than in the ideal case of zero phase noise (i.e., $\phi_{i}^{A}=\phi_{j}^{T}=0$ ), $\boldsymbol{\Phi}_{k}$ has all its entries equal to one, hence, $\hat{\mathbf{L}}_{4}$ and $\hat{\mathbf{C}}_{k}$ become equal to $\mathbf{L}_{4}$ and $\mathbf{C}_{k}$, respectively. We model the phase noises as zero-mean normal random variables each with variance $\sigma_{\phi}^{2}$, and we set as $\sigma_{\phi}^{2}$ the value of the phase noise jitter $0.717^{\circ}$, as computed in [54] for the MAX2829 oscillator. Note that this value has been used in several FD experiments using the analog canceler architecture based on multiple AUX TXs $[14,31]$. We also assume that $\exp \left(j \phi_{i}^{A}\right)$ and $\exp \left(j \phi_{j}^{T}\right) \forall i=1,2, \ldots, N$ and $\forall j=1,2, \ldots, N_{k}$ are IID random variables; this implies that our model considers the case where the TX RF chains have independent oscillators. As was discussed in [54], the amount of analog cancellation for these types of phase noise is approximately $35 \mathrm{~dB}$. This means that the considered multi-AUX-TX canceler architecture in our simulations is capable of delivering approximately $35 \mathrm{~dB}$ of analog cancellation per AUX TX RF chain.

\section{REFERENCES}

[1] "The Next Hyper-Connected Experience for All," White Paper, Samsung 6G Vision, Jun. 2020. 
[2] "Innovations in 5G Backhaul Technologies," White Paper, 5G Americas, Jun. 2020.

[3] A. Sabharwal et al., "In-band full-duplex wireless: Challenges and opportunities," IEEE J. Sel. Areas Commun., vol. 32, no. 9, pp. 1637-1652, Sep. 2014.

[4] T. Riihonen et al., "Mitigation of loopback selfinterference in full-duplex MIMO relays," IEEE Trans. Signal Proces., vol. 59, no. 12, pp. 5983-5993, Dec. 2011.

[5] D. Nguyen et al., "Precoding for full duplex multiuser MIMO systems: Spectral and energy efficiency maximization," IEEE Trans. Signal Process., vol. 61, no. 13, pp. 4038-4050, Aug. 2013.

[6] D. Bharadia and S. Katti, "Full duplex MIMO radios," in Proc. USENIX NSDI, Seattle, WA, 2-4 Apr. 2014, pp. 359-372.

[7] E. Everett et al., "SoftNull: Many-antenna full-duplex wireless via digital beamforming," IEEE Trans. Wireless Commun., vol. 12, no. 15, pp. 8077-8092, Dec. 2016.

[8] H. H. M. Tam et al., "Successive convex quadratic programming for quality-of-service management in fullduplex MU-MIMO multicell networks," IEEE Trans. Commun., vol. 64, no. 6, pp. 2340-2353, Jun. 2016.

[9] I. Atzeni et al., "Performance evaluation of user scheduling for full-duplex small cells in ultra-dense networks," in Proc. Europ. Wireless Conf., Oulu, Finland, 18-20 May 2016, pp. 1-6.

[10] G. C. Alexandropoulos et al., "User scheduling and optimal power allocation for full-duplex cellular networks," in Proc. IEEE SPAWC, Edinburgh, UK, 3-6 Jul. 2016, pp. 1-6.

[11] M. Shafi et al., "5G: A tutorial overview of standards, trials, challenges, deployment, and practice," IEEE J. Sel. Areas Commun., vol. 35, no. 6, pp. 1201-1221, Jun. 2017.

[12] W. Shaad et al., "A vision of 6G wireless systems: Applications, trends, technologies, and open research problems," IEEE Network, vol. 34, no. 3, pp. 134-142, Jun. 2020.

[13] D. Bharadia et al., "Full duplex radios," in Proc. ACM SIGCOMM, Hong Kong, China, 12-16 Aug. 2013, pp. 375-386.

[14] M. Duarte et al., "Design and characterization of a full-duplex multiantenna system for WiFi networks," IEEE Trans. Veh. Technol., vol. 63, no. 3, pp. 11601177, Mar. 2014.

[15] K. E. Kolodziej et al., "Multitap RF canceller for in-band full-duplex wireless communications," IEEE Trans. Wireless Commun., vol. 15, no. 6, pp. 43214334, Jun. 2016.
[16] S. Huberman and T. Le-Ngoc, "MIMO full-duplex precoding: A joint beamforming and self-interference cancellation structure," IEEE Trans. Wireless Commun., vol. 14, no. 4, pp. 2205-2217, Apr. 2015.

[17] G. C. Alexandropoulos and M. Duarte, "Joint design of multi-tap analog cancellation and digital beamforming for reduced complexity full duplex MIMO systems," in Proc. IEEE ICC, Paris, France, 21-25 May 2017, pp. 1-7.

[18] M. Duarte and G. C. Alexandropoulos, "Full duplex MIMO digital beamforming with reduced complexity AUXTX analog cancellation," in Proc. IEEE ICC, Dublin, Ireland, 7-11 June 2020, pp. 1-6.

[19] G. C. Alexandropoulos and M. Duarte, "A fullduplex wireless beamforming apparatus with selfinterference cancellation and method," EPO Patent W02018059691A1, 5 Apr., 2018.

[20] I. Atzeni et al., "Optimal low-complexity selfinterference cancellation for full-duplex MIMO small cells," in Proc. IEEE ICC, Kuala Lumpur, Malaysia, 23-27 May 2016, pp. 1-6.

[21] J. Zhang et al., "Full duplex wireless communications with partial interference cancellation," in Proc. IEEE ASILOMAR, Pasific Grove, USA, 4-7 Nov. 2012, pp. 1295-1299.

[22] N. M. Gowda and A. Sabharwal, "JointNull: Combining partial analog cancellation with transmit beamforming for large-antenna full-duplex wireless systems," IEEE Trans. Wireless Commun., vol. 17, no. 3, pp. 2094-2108, Mar. 2018.

[23] M. A. Islam et al., "Joint analog and digital transceiver design for wideband full duplex MIMO systems," IEEE Trans. Wireless Commun., under revision, 2021.

[24] D. Korpi et al., "Nonlinear self-interference cancelllation in MIMO full-duplex transceivers under crosstalk," EURASIP J. Wireless Commun. Netw., vol. 24, no. 1, pp. 1-15, Dec. 2017.

[25] M. A. Islam et al., "A unified beamforming and A/D self-interference cancellation design for full duplex MIMO radios," in Proc. IEEE PIMRC, Istanbul, Turkey, 8-11 September 2019, pp. 1-6.

[26] E. Björnson et al., "Multiobjective signal processing optimization," IEEE Signal Process. Mag., vol. 31, no. 6, pp. 14-23, Nov. 2014.

[27] H. Huang et al., MIMO Communication for Cellular Networks. New York, USA: Springer, 2012.

[28] E. Telatar, "Capacity of multi-antenna Gaussian channels," European Trans. Telecommun., vol. 10, no. 6, pp. 585-595, Dec. 1999. 
[29] G. C. Alexandropoulos and C. B. Papadias, "A reconfigurable iterative algorithm for the $K$ - user MIMO interference channel," Signal Process. (Elsevier), vol. 93, no. 12, pp. 3353-3362, Dec. 2013.

[30] M. Sadek et al., "Active antenna selection in multiuser MIMO communications," IEEE Trans. Signal Process., vol. 4, no. 55, pp. 1498-1510, Apr. 2007.

[31] M. Duarte et al., "Experiment-driven characterization of full-duplex wireless systems," IEEE Trans. Wireless Commun., vol. 11, no. 12, pp. 4296-4307, Dec. 2012.

[32] P. Joshi et al., "Output power levels of 4G user equipment and implications on realistic RF EMF exposure assessments," IEEE Access, vol. 5, pp. 4545-4550, Mar. 2017.

[33] A. F. Molisch et al., "Hybrid beamforming for massive MIMO: A survey," IEEE Commun. Mag., vol. 55, no. 9, pp. 134-141, Sep. 2017.

[34] H. Iimori et al., "MIMO beamforming schemes for hybrid SIC FD radios with imperfect hardware and CSI," IEEE Trans. Wireless Commun., vol. 18, no. 10, pp. 4816-4830, Oct. 2019.

[35] G. C. Alexandropoulos et al., "Full duplex hybrid A/D beamforming with reduced complexity multi-tap analog cancellation," in Proc. IEEE SPAWC, Atlanta, USA, 26-29 May 2020, pp. 1-6.

[36] A. T. Le et al., "Beam-based analog self-interference cancellation in full-duplex MIMO systems," IEEE Trans. Wireless Commun., vol. 19, no. 4, pp. 24602471, Apr. 2020.

[37] X. Huang et al., "Transmit beamforming for communication and self-interference cancellation in fullduplex MIMO systems: A trade-off analysis," IEEE Trans. Wireless Commun., vol. 20, no. 6, pp. 37603769, Jun. 2021.

[38] A. T. Le et al., "Analog least mean square adaptive filtering for self-interference cancellation in full duplex radios," IEEE Wireless Commun., vol. 28, no. 1, pp. 12-18, Feb. 2021.

[39] M. A. Islam et al., "Simultaneous downlink data transmission and uplink channel estimation with reduced complexity full duplex MIMO radios," in Proc. IEEE ICC, Dublin, Ireland, 7-11 June 2020, pp. 1-6.

[40] M. A. Islam et al., "Simultaneous data communication and channel estimation in multiuser full duplex MIMO systems," in Proc. IEEE Asilomar SSCC, Pacific Grove, USA, 1-5 November 2020, pp. 1-5.

[41] M. A. Islam et al., "Direction-assisted beam management in full duplex millimeter wave massive MIMO systems," in Proc. IEEE GLOBECOM, Madrid, Spain, 7-11 December 2021, pp. 1-6.
[42] C. B. Barneto et al., "Full duplex radio/radar technology: The enabler for advanced joint communication and sensing," IEEE Wireless Commun., vol. 28, no. 1, pp. 82-88, Feb. 2021.

[43] M. A. Islam et al., "Integrated sensing and communication with millimeter wave full duplex hybrid beamforming," IEEE ICC, Seoul, South Korea, 16-20 May 2022, under review.

[44] C. Zhang et al., "Deep learning in mobile and wireless networking," IEEE Commun. Surveys Tuts., vol. 21, no. 3, pp. 2224-2287, 2019.

[45] C. Huang et al., "Deep learning for UL/DL channel calibration in generic massive MIMO systems" in Proc. IEEE ICC, Shanghai, China, 20-24 May 2019, pp. 1-6.

[46] G. C. Alexandropoulos et al., "Pervasive machine learning for smart radio environments enabled by reconfigurable intelligent surfaces" Proc. IEEE, 2021, under review.

[47] M. Di Renzo et al., "Smart radio environments empowered by AI reconfigurable meta-surfaces: An idea whose time has come," EURASIP J. Wireless Commun. Netw., 129, pp. 1-20, May 2019.

[48] E. Calvanese Strinati et al., "Wireless environment as a service enabled by reconfigurable intelligent surfaces: The RISE-6G perspective," in Proc. Joint EuCNC \& 6G Summit, Porto, Portugal, 8-11 June 2021, pp. 1-6.

[49] E. Calvanese Strinati et al., "Reconfigurable, intelligent, and sustainable wireless environments for $6 \mathrm{G}$ smart connectivity," IEEE Commun. Mag., to appear, 2021.

[50] C. Huang et al., "Reconfigurable intelligent surfaces for energy efficiency in wireless communication," IEEE Trans. Wireless Commun., vol. 18, no. 8, pp. 4157-4170, Aug. 2019.

[51] G. C. Alexandropoulos et al., "Reconfigurable intelligent surfaces for rich scattering wireless communications: Recent experiments, challenges, and opportunities," IEEE Commun. Mag., vol. 59, no. 6, pp. 28-34, Jun. 2021.

[52] I. Alamzadeh et al., "A reconfigurable intelligent surface with integrated sensing capability," Sci. Rep., vol. 11, no. 1, pp. 1-10, 2021.

[53] N. Shlezinger et al., "Dynamic metasurface antennas for 6G extreme massive MIMO communications," IEEE Wireless Commun., vol. 28, no. 2, pp. 106-113, Apr. 2021.

[54] A. Sahai et al., "On the impact of phase noise on active cancelation in wireless full-duplex," IEEE Trans. Veh. Technol., vol. 62, no. 9, pp. 4494-4510, Nov. 2013. 


\section{AUTHOR}

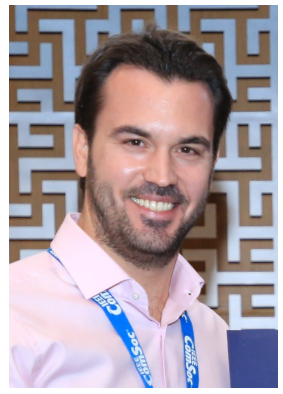

George C. Alexandropoulos received his Engineering Diploma, M.A.Sc., and Ph.D. degrees in Computer Engineering and Informatics from the University of Patras, Greece in 2003, 2005, and 2010, respectively. He has held research positions at various Greek universities and research institutes (namely, National Center for Scientific Research "Demokritos," National Observatory of Athens, Institute of Accelerating Systems and Applications, Athens Information Technology (AIT) Center for Research and Education, Telecommunication Systems Research Institute of the Technical University of Crete, and Universities of Patras and Peloponnese), as well as at the Mathematical and Algorithmic Sciences Lab, Paris Research Center, Huawei Technologies France, and he is currently an Assistant Professor for Wireless Communication Systems and Signal Processing with the Department of Informatics and Telecommunications, National and Kapodistrian University of Athens (NKUA), Greece. His research interests span the general areas of algorithmic design and performance analysis for wireless networks with emphasis on multi-antenna transceiver hardware architectures, full duplex radios, active and passive reconfigurable metasurfaces, millimeter wave communications, as well as distributed machine learning algorithms. He currently serves as an Editor for IEEE Transactions on Wireless Communications, IEEE Communications Letters, IEEE Wireless Communications Letters, ELSEVIER Computer Networks, Frontiers in Communications and Networks, and the ITU Journal on Future and Evolving Technologies, as well as a guest editor for various IEEE special issues. He has received the best Ph.D. thesis award 2010, the IEEE Communications Society Best Young Professional in Industry Award 2018, the EURASIP Best Paper Award of the Journal on Wireless Communications and Networking 2021, and the IEEE Marconi Prize Paper Award in Wireless Communications 2021. Prof. Alexandropoulos is a Senior Member of IEEE and the IEEE Communications, Signal Processing, and Information Theory Societies, as well as a registered Professional Engineer at the Technical Chamber of Greece. He has organized various special sessions and workshops in flagship conferences of the latter IEEE societies, where he also delivered various tutorials and invited talks on his fields of interest. He has participated and/or technically managed more than 10 European Union (EU) research and innovation projects, as well as several Greek and international research projects. He is currently NKUA's principal investigator for the EU H2020 RISE-6G project under grant number 101017011. More information is available at www.alexandropoulos.info. 